\title{
Discretization Errors in the Hybrid Finite Element Particle-in-cell Method
}

\section{Journal Article}

\section{Author(s):}

Thielmann, Marcel; May, Dave A.; Kaus, Boris J.P.

Publication date:

2014-09

\section{Permanent link:}

https://doi.org/10.3929/ethz-b-000089955

\section{Rights / license:}

In Copyright - Non-Commercial Use Permitted

\section{Originally published in:}

Pure and Applied Geophysics 171(9), https://doi.org/10.1007/s00024-014-0808-9 


\title{
Discretization Errors in the Hybrid Finite Element Particle-in-cell Method
}

\author{
M. Thielmann, ${ }^{1,2}$ D. A. May, ${ }^{1}$ and B. J. P. Kaus ${ }^{3}$
}

\begin{abstract}
In computational geodynamics, the Finite Element (FE) method is frequently used. The method is attractive as it easily allows employment of body-fitted deformable meshes and a true free surface boundary condition. However, when a Lagrangian mesh is used, remeshing becomes necessary at large strains to avoid numerical inaccuracies (or even wrong results) due to severely distorted elements. For this reason, the FE method is oftentimes combined with the particle-in-cell (PIC) method, where particles are introduced which track history variables and store constitutive information. This implies that the respective material properties have to be interpolated from the particles to the integration points of the finite elements. In numerical geodynamics, material parameters (in particular the viscosity) usually vary over a large range. This may be due to strongly temperature-dependent rheologies (which result in large but smooth viscosity variations) or material interfaces (which result in viscosity jumps). Here, we analyze the accuracy and convergence properties of velocity and pressure of the hybrid FE-PIC method in the presence of large viscosity variations. Standard interpolation schemes (arithmetic and harmonic) are compared to a more sophisticated interpolation scheme which is based on linear least squares interpolation for two types of elements $\left(Q_{1} P_{0}\right.$ and $\left.Q_{2} P_{-1}\right)$. In the case of a smooth viscosity field, the accuracy and convergence is significantly improved by the new interpolation scheme. In the presence of viscosity jumps, the order of accuracy is strongly decreased.
\end{abstract}

Key words: Numerical modeling, Particle-in-cell, Finite element method, Interpolation.

\section{Introduction}

\subsection{Background}

In the last two decades, numerical models have increasingly become a valuable tool to grasp the

\footnotetext{
1 Institut f. Geophysik, ETZ Zürich, Sonneggstrasse 5, 8092 Zurich, Switzerland. E-mail: marcel.thielmann@erdw.ethz.ch

2 Institute for Building Materials, ETH Zürich, Schafmattstr. 6, 8093 Zurich, Switzerland.

3 Institute of Geosciences, Center for Computational Sciences \& Center for Volcanoes, Atmospheres and Magmatic Open Systems, Johannes Gutenberg-Universität Mainz, J.-J.-Becher-Weg 21, 55099 Mainz, Germany.
}

underlying physics of geological processes ranging from mantle convection to grain-scale processes. However, geodynamic processes entail a number of physical characteristics, which are challenging from a numerical modelling perspective.

The deformation of Earth materials is commonly governed by three major rheologies: elastic, viscous and plastic deformation. Viscous deformation can be accommodated through a variety of solid-state creep mechanisms, ranging from (grain size sensitive) diffusion creep over dislocation creep to highly nonlinear Peierls creep. At low pressures and sufficiently high stresses, brittle failure (also being highly nonlinear) dominates. Additionally, elasticity significantly influences deformation at low temperatures and on short timescales. The rheologies describing the different deformation mechanisms are also strongly dependent on the chemistry of the deforming rock.

The Earth's mantle and lithosphere are strongly heterogeneous. Numerical codes, thus, have to be able to accurately track and advect material properties and history variables (temperature, grain size, chemistry, damage, etc.) throughout the model. Faults-brittle or ductile-in the lithosphere represent sharp contrasts in effective viscosities. In Fig. 1 we show the viscosity field and the viscosity contrast within a finite element of a typical 'realistic' subduction simulation. Subduction is initiated using a weak zone (weak in the sense that is has a low cohesion (1 MPa) and a zero friction angle) that decouple the two adjacent oceanic plates (which consist of an upper crust, a lower crust and a lithospheric mantle). The rheology is viscoplastic with a power-law rheology for the viscous deformation (with olivine parameters in the mantle) and plastic deformation is modeled using a DruckerPrager yield criterion (the setup was inspired, but not identical to the one used in Quinquis and BuITER 


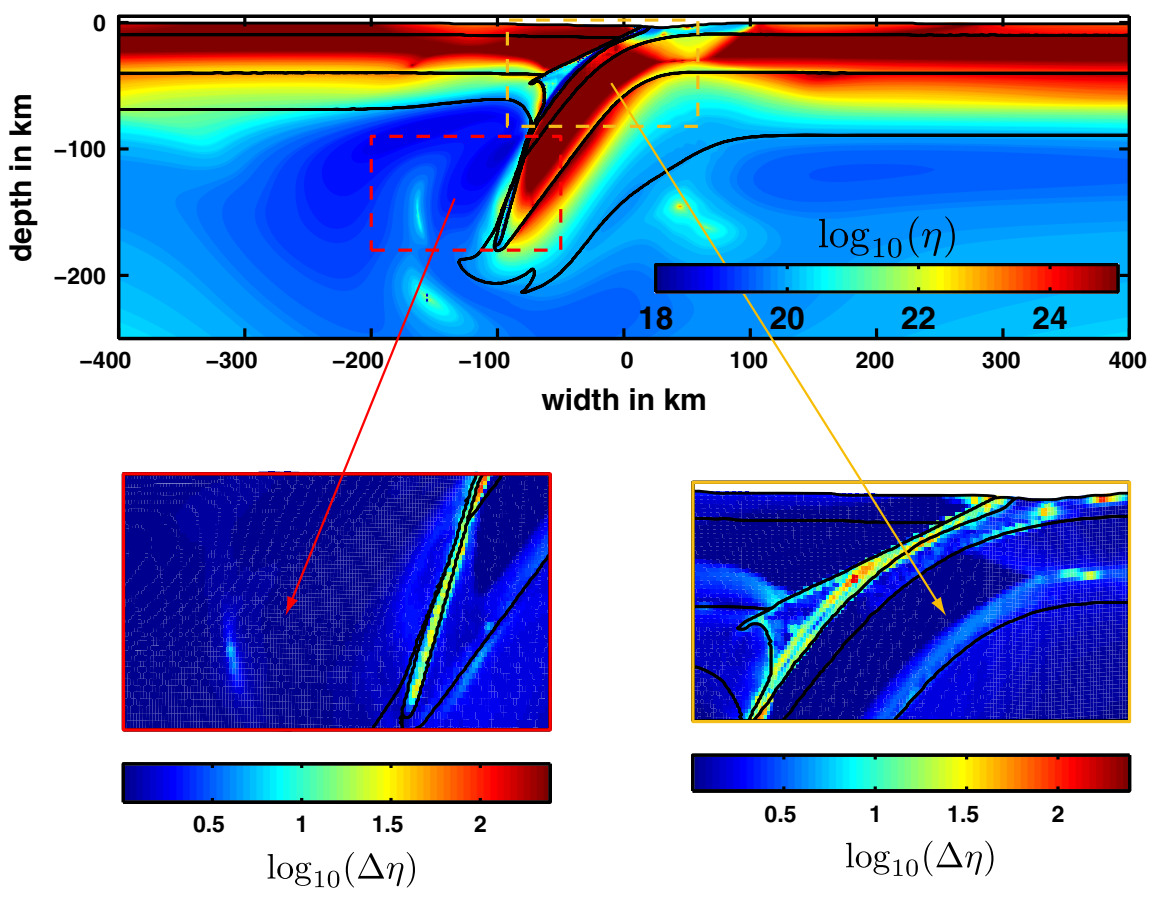

Figure 1

Viscosity fields and viscosity contrasts in 'realistic' mantle-lithosphere models. In the top row, the viscosity field of a typical subduction simulation is shown. Dashed boxes mark the location of two zoomed in regions where viscosity contrasts per element are plotted (bottom row). Solid black lines denote boundaries between different lithological layers

2013). Resolution is $1,201 \times 401$ nodes. In this example $Q_{2} P_{-1}$ elements were employed. One can clearly see that the highest viscosity contrasts occur close to the surface and in the weak zone, which is due to plastic failure. In regions where ductile creep is dominant, viscosity contrasts are much lower, and often less than a factor 6 .

It has been shown that variations in viscosity within a single element can lead to a significant decrease in the quality of the numerical solution (Mackinnon and CARey 1987; Moresi et al. 1996). Furthermore, Moresi et al. (1996) showed that the accuracy of the numerical solution can directly depends on the magnitude of the variation of viscosity within an element. Additionally, the convergence of numerical solvers can significantly decrease when the viscosity fields exhibit jumps (e.g., Albers 2000; May and Moresi 2008; Tackley 2000; TROMPERT and HANSEN 1996). Therefore, if one wants to obtain accurate solutions for problems with strong and sharp viscosity contrasts, it is advisable to use an unstructured grid together with higher order shape functions (Deubelbeiss and Kaus 2008) designed such that element boundaries conform to the jumps in viscosity.

When studying processes over million year time scales, the material will undergo severe mixing and experience extremely high strains (large deformation). In this regime, using body fitted methods becomes becomes unfeasible. Hence, it is therefore necessary to develop alternative methods which can still track sharp variations in material properties in a large deformation regime without requiring unstructured, body fitted meshes.

To simulate geological processes, it is typically required that (1) we can represent compositionally distinct materials (e.g., interface tracking) and (2) within each composition, we can track the evolution of volumetric history variables such as accumulated plastic strain, or elasticity. The evolution of the material interfaces and history variables are given by an advection equation (e.g., no diffusion is present).

Excluding using body fitted meshes, which follow the evolution in a Lagrangian manner, there are several 
approaches to represent interfaces. Interfaces can be described using a scalar quantity $f$, where $f=0, f=1$ denote the regions on either side of the interface and the iso-surface $f=\frac{1}{2}$ denotes the interface. The classical Level sets method (Osher and FedKiw 2003; Sethian 1999) provides an equivalent, yet more compact representation, compared to the field method as it describes the interface in a lower spatial dimensional. Whilst both methods enable topologically complex interface geometries to be represented, they both require an accurate grid based advection solver.

Accurate grid based advection solvers can be constructed using high order finite volume (FV) discretisations combined with flux limiters (TVD, ENO, WENO, etc) (LeVeque 2002). Similarly, high order (in space and time) discontinuous Galerkin (DG) discretisations (Cockburn and SHu 1998; CockBURn et al. 2000) have also proven to be robust for pure advection equations. Whilst those methods are sufficiently accurate and are suitable for evolving equations associated interface and history variable evolution, their implementation is involved and the methods are computationally expensive. Moreover, it is not straightforward to implement such methods in the case where phase transitions occur, or where jumps in viscosity develop spontaneously during a simulation once shear bands form. Yet, this is a situation that is quite typical in numerical simulations of lithosphere dynamics.

Another possibility to represent material interfaces, which avoids the need for a grid based advection solver, is the marker-chain method ( $\mathrm{V}_{\mathrm{AN}}$ KeKEN et al. 1997). When large strain deformation occurs, this method may break down due to overlapping marker-chains. The method is also non-trivial to implement in 3D (although see Kaus and PodladCHIKOV 2001 for a geodynamic application). In addition, the marker-chain method does not provide a mechanism to track history variables within the respective material domains.

An elegant solution, which allows one to represent compositionally distinct materials and track history dependent information, is to employ "particle methods" in which the material properties are represented by a set of independent Lagrangian points. Advection of composition (volumetric representation of the interface) and history variables is achieved by simply updating the particles position-thus, these methods do not suffer from numerical difficulties associated with grid based equation solvers. Furthermore, there is no assumed connectivity between particles, thus, topologically complex material interfaces in $3 \mathrm{D}$ can be trivially represented. The robustness and ease of their implementation (particularly in 3D) have made particle methods popular within the geodynamics community (e.g., Gerya 2003; Moresi et al. 2003; Poliakov and Podladchikov 1992; TACKLey and King 2003). Despite the wide spread usage of the particle method, few studies have provided a detailed analysis of the accuracy of this method (Deubelbeiss and Kaus, 2008; DuRETZ et al. 2011).

Despite the advantages of the hybrid methods, there are several issues concerning their accuracy. Solving the governing equations on a grid and storing the material properties on particles requires interpolating various physical quantities between the particles and the grid points. The accuracy of this interpolation step might depend on both the number of particles per cell and the interpolation scheme used. Deubelbeiss and Kaus (2008) analyzed how both the spatial discretisation used to solve the variable viscosity Stokes problem and the interpolation technique (arithmetic, harmonic or geometric interpolation) applied to either viscosity or density influenced the numerical accuracy of the velocity and pressure field. They found that the choice of the "best" averaging method is both dependent on the physical problem and the physical parameter (e.g., viscosity or density) which has to be interpolated. DURETZ et al. (2011) conducted a study where they investigated the convergence properties of the interpolation method used in GerYa (2003). They found that the accuracy of the numerical solution can be significantly increased when using a more local support for the interpolation in combination with staggered finite difference methods .

\subsection{Motivation}

Finite element methods have been widely used to model mantle and lithosphere dynamics by using a continuum description which resembles a steady state, incompressible variable viscosity Stokes formulation. In considering geodynamic applications, 
we note that the exterior boundary of the modeling domain (e.g., the sphere of the Earth) is relatively simple. Furthermore, even with the inclusion of a free surface or a landscape evolution model, the resulting topography development does not create a complex exterior boundary. Thus, here we use regular meshes with quadrilateral elements to discretize the geometry of the domain. Internal geometric complexities will be represented using a particle-in-cell (PIC) scheme.

In the finite element approach, the weak form of the governing equations is developed by discretizing the model domain into a set of non-overlapping elements. Within each element, the unknown quantities are approximated via locally defined interpolants (or shape functions). The weak formulation involves the evaluation of integrals defined element wise. These integrals are approximated via numerical quadrature, e.g., Gauss-Legendre (e.g., ZiENKIEwICZ et al. 2005). For such quadrature schemes, it is necessary to define a set of points where the integrand will be evaluated and integration weights which will then be used in the quadrature summation. In hybrid finite-element-particle-in-cell schemes (FE-PIC), it is required to interpolate the needed properties from the particles to the quadrature points. Interpolation methods like the ones used in (Deubelbeiss and Kaus 2008) result in the prescription of a single value for a given property on all quadrature points in an element. However, the finite element method permits material parameters to vary within the element, thus, resulting in a better spatial representation of material parameters. Using interpolation methods that prescribe one single value to all quadrature points, therefore, does not exploit the full potential of the method and might unnecessarily limit its accuracy. For this reason, we here introduce an interpolation method which allows properties to vary throughout the element and investigate the error and order of accuracy of the hybrid FE-PIC numerical method. Accuracy is assessed by comparing analytic solutions to variable viscosity Stokes flow to numerical experiments using different marker and grid resolution, as well as differing interpolation schemes. In this study, we use the code MILAMIN_VEP (e.g., KAUs et al. 2010), which is based on MILAMIN developed by DABRowsKi et al. (2008), but is extended by remeshing, visco-elasto-plastic rheologies as well as a particle-based method to advect material properties. Here, we focus on the effect of the particle-based method on both the accuracy and convergence rate of the numerical solution of three idealized geodynamic model configurations for which analytical solutions exist.

\section{Governing Equations}

In geodynamics, the deformation of rocks over long time spans is (in its simplest form) described via the incompressible Stokes equations. The conservation of mass and momentum is given by

$$
\begin{gathered}
\frac{\partial v_{i}}{\partial x_{i}}=0 \\
-\frac{\partial p}{\partial x_{i}}+\frac{\partial \tau_{i j}}{\partial x_{j}}=-\rho g_{i}
\end{gathered}
$$

where $v_{i}$ denotes velocity, $p$ pressure, $\tau_{i j}$ the deviatoric stress tensor and $g_{i}$ gravitational acceleration. In the case of an isotropic, linear viscous material, the deviatoric stress tensor can be related to the strain rate tensor $\dot{\varepsilon}_{i j}$ using

$$
\tau_{i j}=2 \eta \dot{\varepsilon}_{i j}
$$

where $\eta$ is the shear viscosity and $\dot{\varepsilon}_{i j}$ is defined as

$$
\dot{\varepsilon}_{i j}=\frac{1}{2}\left(\frac{\partial v_{i}}{\partial x_{j}}+\frac{\partial v_{j}}{\partial x_{i}}\right) .
$$

Whilst there are no time derivatives in the Stokes equations, the coefficients $(\eta, \rho)$ evolve according to

$$
\frac{D \eta}{D t}=0, \quad \frac{D \rho}{D t}=0,
$$

thereby introducing a temporal dependency.

\section{Numerical Method}

We solve the governing Eqs. (1) and (2) expressed in the primitive variables $v_{i}, p$, using a mixed finite element discretization. Here we use two types of quadrilateral elements: $Q_{1} P_{0}$ elements with linear shape functions for velocity and a constant shape function for pressure and $Q_{2} P_{-1}$ elements with quadratic shape functions for velocity and linear 
discontinuous shape functions for pressure. Evaluation of the element bilinear form for the viscous stress gradient and linear form for the forcing function are approximated via numerical quadrature, i.e.,

$$
\int_{\Omega_{e}} \eta \hat{\dot{\varepsilon}}_{i j} \hat{\dot{\varepsilon}}_{i j} d V \approx \sum_{q} w_{q} \eta\left(\xi_{q}\right) \hat{\dot{\varepsilon}}_{i j}\left(\xi_{q}\right) \hat{\dot{\varepsilon}}_{i j}\left(\xi_{q}\right) \operatorname{det} \mathbf{J}\left(\xi_{\mathbf{q}}\right)
$$

and

$$
\int_{\Omega_{e}} N \rho g_{i} d V \approx \sum_{q} w_{q} N\left(\xi_{q}\right) \rho\left(\xi_{q}\right) g_{i} \operatorname{det} \mathbf{J}\left(\xi_{\mathbf{q}}\right),
$$

where $\Omega_{e}$ is the element domain, $\hat{\dot{\varepsilon}}_{i j}$ is the discrete strain rate operator, $N$ is the basis function chosen for $v_{i}, \mathbf{J}$ is the coordinate transformation between $\Omega_{e}$ and the reference element and $\xi_{q}$ is the local coordinate of the $q$ th quadrature point with weight $w_{q}$. Lagrangian markers are employed to store the material parameters (density $\rho$, viscosity $\eta$ ) of different rock types. The marker properties are then used to define the quadrature points values $\rho\left(\xi_{q}\right), \eta\left(\xi_{q}\right)$ within each element $\Omega_{e}$ via an interpolation procedure.

\subsection{Piece-wise Constant Interpolation}

Many numerical codes used in the geodynamical community employ a particle-in-cell scheme, where lithological properties are stored on a set of Lagrangian markers. The material property $\Phi$ has to be projected onto the quadrature points used to evaluate the weak form associated with the finite element formulation. A number of piece-wise constants (over each element $\Omega_{e}$ ) with interpolation schemes can be defined. Two of the most common schemes are:

- Arithmetic average:

$$
\Phi_{e}=\frac{\sum_{p} \Phi_{p}}{\sum_{p} 1}
$$

- Harmonic average:

$$
\Phi_{e}=\frac{\sum_{p} 1}{\sum_{p} \Phi_{p}^{-1}},
$$

where $p$ is the index of each particle located within inside element $e$. The value of $\Phi$ assigned to each quadrature point with element $e$ is simply $\Phi\left(\xi_{q}\right)=\Phi_{e}$. Note that both interpolation schemes define a constant value of $\Phi$ over the entire element. Thus, the spatial variation of material parameters inside the element is absent. Commonly, arithmetic averaging is used for density interpolation. The physical meaning of those two averaging schemeswhen used for viscosity averaging-has been discussed in Schmeling et al. (2008). The main points are that harmonic averaging corresponds to a rheological model where two viscous elements are in series. This model correctly describes the volume-averaged effective viscosity in a situation where the compositional interface is aligned parallel to the flow (e.g., channel flow or simple shear), where the effective viscosity is governed by the weaker of the two viscosities. Arithmetic averaging, on the other hand, corresponds to two viscous elements in parallel, thus, the stronger viscosity will dominate the effective viscosity. This situation can be found in regions where the interface is subjected to interface-parallel shear.

\subsection{Least Squares Interpolation}

Here we introduce an element-wise interpolation scheme based on a least squares fitting of the particle properties. Denoting the physical quantity by $\Phi(\mathbf{x})$, we will assume that $\Phi$ is sampled at $n$ locations with spatial coordinates $\left(x_{1} \ldots x_{n}, y_{1} \ldots y_{n}\right)$ with values $\Phi_{1} \ldots \Phi_{n}$. If $\Phi(\mathbf{x})$ is approximated via a function $\tilde{\Phi}(\mathbf{x})$, the squared error $\epsilon^{2}$ between $\tilde{\Phi}(\mathbf{x})$ and $\Phi(\mathbf{x})$ is given by:

$$
\epsilon^{2}=\sum_{i=1}^{n}\left[\tilde{\Phi}(x, y)-\Phi_{i}\right]^{2} .
$$

The minimum of $\epsilon^{2}$ can be found where $\nabla\left(\epsilon^{2}\right)=0$. Here we choose to use a linear function,

$$
\Phi(\mathbf{x}) \approx \tilde{\Phi}(\mathbf{x})=c_{3}+c_{1} x+c_{2} y,
$$

to approximate $\Phi(\mathbf{x})$ for two reasons: (1) the mixed elements used in this study employ (at most) a linear pressure shape function and (2) the least squares fitting procedure for this order polynomial is relatively cheap to implement. For a linear function $\nabla\left(\epsilon^{2}\right)$ is defined via

$$
2 \sum_{i=1}^{n}\left[\left(c_{1} x_{i}+c_{2} y_{i}+c_{3}\right)-\Phi_{i}\right]\left(x_{i}, y_{i}, 1\right)=(0,0,0) \text {. }
$$


The coefficients $c_{1}, c_{2}$ and $c_{3}$ can be obtained by solving the following linear system of equations

$$
\begin{aligned}
\underbrace{\left(\begin{array}{ccc}
\sum_{i}^{n} x_{i}^{2} & \sum_{i=1}^{n} x_{i} y_{i} & \sum_{i=1}^{n} x_{i} \\
\sum_{i=1}^{n} x_{i} y_{i} & \sum_{i=1}^{n} y_{i}^{2} & \sum_{i=1}^{n} y_{i} \\
\sum_{i=1}^{n} x_{i} & \sum_{i=1}^{n} y_{i} & \sum_{i=1}^{n} 1
\end{array}\right)}_{\mathbf{A}} \underbrace{\left(\begin{array}{l}
c_{1} \\
c_{2} \\
c_{3}
\end{array}\right)}_{\mathbf{c}} \\
=\underbrace{\left(\begin{array}{c}
\sum_{i=1}^{n} \Phi_{i} x_{i} \\
\sum_{i=1}^{n} \Phi_{i} y_{i} \\
\sum_{i=1}^{n} \Phi_{i}
\end{array}\right)}_{\mathbf{r}} .
\end{aligned}
$$

where we define $\mathbf{A}$ as the coefficient matrix, $\mathbf{c}$ as the solution vector containing the coefficients of the fitted function and $\mathbf{r}$ as the right hand side vector. In the case when $\tilde{\Phi}$ is a linear function (where we would have to invert a $3 \times 3$ matrix), we can use Cramer's rule to obtain the solution vector $\mathbf{c}$, thus, we have
We define this interpolation scheme as "elementwise" as we perform an independent least square fit for $\eta, \rho$ within each element, using only the particles located within that element. Once the particles within each element have been identified, the coefficients for the element $c_{1}, c_{2}, c_{3}$ can be directly computed using (16). Using (11), the value of $\tilde{\Phi}$ at each quadrature point within the element can be evaluated.

\subsubsection{Over- and Under-shooting}

If $\Phi(\mathbf{x})$ possesses a jump inside an element, the value of $\tilde{\Phi}$ at both the quadrature points and the nodes might be significantly larger or smaller than the maximum or minimum value of the same physical property on the particles. This over-shooting or under-shooting is not desirable as it might have a severe impact on the solution. In extreme cases, even

$$
\mathbf{A}^{-1} \mathbf{r}=\frac{1}{\operatorname{det}(\mathbf{A})}\left(\begin{array}{ccc}
a_{22} a_{33}-a_{23}^{2} & a_{13} a_{23}-a_{12} a_{33} & a_{12} a_{23}-a_{13} a_{22} \\
a_{23} a_{13}-a_{12} a_{33} & a_{11} a_{33}-a_{13}{ }^{2} & a_{12} a_{13}-a_{11} a_{23} \\
a_{12} a_{23}-a_{13} a_{22} & a_{12} a_{13}-a_{11} a_{23} & a_{11} a_{22}-a_{12}
\end{array}\right)\left(\begin{array}{c}
r_{1} \\
r_{2} \\
r_{3}
\end{array}\right)
$$

where $\operatorname{det}(\mathbf{A})$ can be computed using the rule of Sarrus, i.e.,

$$
\begin{aligned}
\operatorname{det}(\mathbf{A})= & a_{11} a_{22} a_{33}+2 a_{12} a_{13} a_{23}-a_{13}{ }^{2} a_{22}-a_{11} a_{23}{ }^{2} \\
& -a_{12}{ }^{2} a_{33} .
\end{aligned}
$$

The coefficients $c_{1}, c_{2}, c_{3}$ are given by: unphysical results are possible (e.g., negative viscosities). In our implementation of the linear least squares interpolation, we, therefore, check each element for over-shooting and under-shooting by inspecting the value of $\tilde{\Phi}$ at the nodes of each element. We choose to use the nodal values as those to represent the locations where over-shooting and

$$
\begin{aligned}
& c_{1}=\frac{\left(a_{22} a_{33}-a_{23}^{2}\right) r_{1}+\left(a_{13} a_{23}-a_{12} a_{33}\right) r_{2}+\left(a_{12} a_{23}-a_{13} a_{22}\right) r_{3}}{a_{11} a_{22} a_{33}+2 a_{12} a_{13} a_{23}-a_{13}^{2} a_{22}-a_{11} a_{23}^{2}-a_{12}^{2} a_{33}}, \\
& c_{2}=\frac{\left(a_{23} a_{13}-a_{12} a_{33}\right) r_{1}+\left(a_{11} a_{33}-a_{13}{ }^{2}\right) r_{2}+\left(a_{12} a_{13}-a_{11} a_{23}\right) r_{3}}{a_{11} a_{22} a_{33}+2 a_{12} a_{13} a_{23}-a_{13} a_{22}-a_{11} a_{23}{ }^{2}-a_{12} a_{33}}, \\
& c_{3}=\frac{\left(a_{12} a_{23}-a_{13} a_{22}\right) r_{1}+\left(a_{12} a_{13}-a_{11} a_{23}\right) r_{2}+\left(a_{11} a_{22}-a_{12}\right) r_{3}}{a_{11} a_{22} a_{33}+2 a_{12} a_{13} a_{23}-a_{13}^{2} a_{22}-a_{11} a_{23}{ }^{2}-a_{12} a_{33}}
\end{aligned}
$$


under-shooting have the biggest effect. Additionally, as we expect both the density and viscosity field to be continuous, the values at the nodes should not exhibit jumps that are too large. The nodal values that exhibit smaller/larger values than the minimum/maximum values stored on the particles are then set to the respective minimum and maximum values. We then compute the new coefficients of the linear regression plane using the updated nodal values only. This procedure is repeated until no nodal values are overshooting or under-shooting. The values at the quadrature points use the corrected coefficients. For better convergence of this iterative algorithm, we slightly relax the upper and lower limits and allow nodal values to over-/under-shoot by $1 \%$ of the respective maximum/minimum value.

\section{Test Cases}

The scheme used to interpolate $\eta$ or $\rho$ should be able to deal with several situations: (1) it should be able to approximate a smoothly varying density and/ or viscosity field, and (2) it should be able to deal with jumps in both fields. To compare the performance of the different interpolation algorithms, we conduct the same three benchmark tests as DuRETZ et al. (2011). The first test addresses buoyancy-driven flow in the presence of large but smooth viscosity variations [see Revenaugh and Parsons (1987), termed SolKz by DuRETz et al. (2011)] whereas the second test employs a viscosity jump in the middle of the domain [see (Moresi et al. 1996), termed SolCx by Duretz et al. (2011)]. The third test consists of a weak matrix with a highly viscous circular inclusion that is deformed in pure shear (Schmid 2003) (SolVI). In the first two cases, flow is driven by an internal force, which-in the case of geodynamic applications-can be interpreted as arising due to buoyancy differences. Consequently, we express the forcing term in those experiments in terms of a density field $\rho$ and a constant gravity field. Here, gravity equals zero in the $x$-direction and -1 in the $y$-direction.

\subsection{Smooth Viscosity Variation in One Dimension}

The analytical solution of Revenaugh and ParSONS (1987) allows us to investigate the accuracy of our interpolation schemes for the case of smoothly varying density and viscosity distributions. The model domain is a box of height and width one with free slip boundary conditions employed on all sides. The variation of viscosity with depth is given by

$$
\eta(x, y)=\exp (2 B y)
$$

where $B$ is chosen in such a way that the viscosity ratio between top and bottom equals $10^{6}$. Density is prescribed as

$$
\rho(x, y)=-\sin (3 \pi x) \cos (2 y) .
$$

The viscosity and density fields are shown in Fig. 2 together with the resulting velocity, pressure and stress fields. The $\mathrm{C}$ source code for this analytic solution is available from the open source package Underworld (MORESI et al. 2007).

\subsection{SolCx: Viscosity Jump in One Dimension}

The SolCx benchmark again consists of a $1 \times 1$ box with free slip boundaries on all sides. Contrary to the SolKz benchmark, only the density distribution is smoothly varying and the viscosity distribution is characterized by a viscosity jump at $x_{c}=0.5$ (see Fig. 3). This test is somewhat harder than the SolKz test, as the viscosity jump is very hard to capture using any interpolation scheme. The complete derivation of the analytical solution is described in ZHONG (1996). The viscosity field employed in this study is described by

$$
\eta(x, y)=\left\{\begin{array}{ccc}
1, & \text { if } & x_{\mathrm{c}} \leq 0.5 \\
10^{3}, & \text { if } & x_{\mathrm{c}}>0.5
\end{array} .\right.
$$

and the density via

$$
\rho(x, y)=\sin (\pi y) \cos (\pi x) .
$$

The C source code for this analytic solution is available from the open source package Underworld (MORESI et al. 2007). 

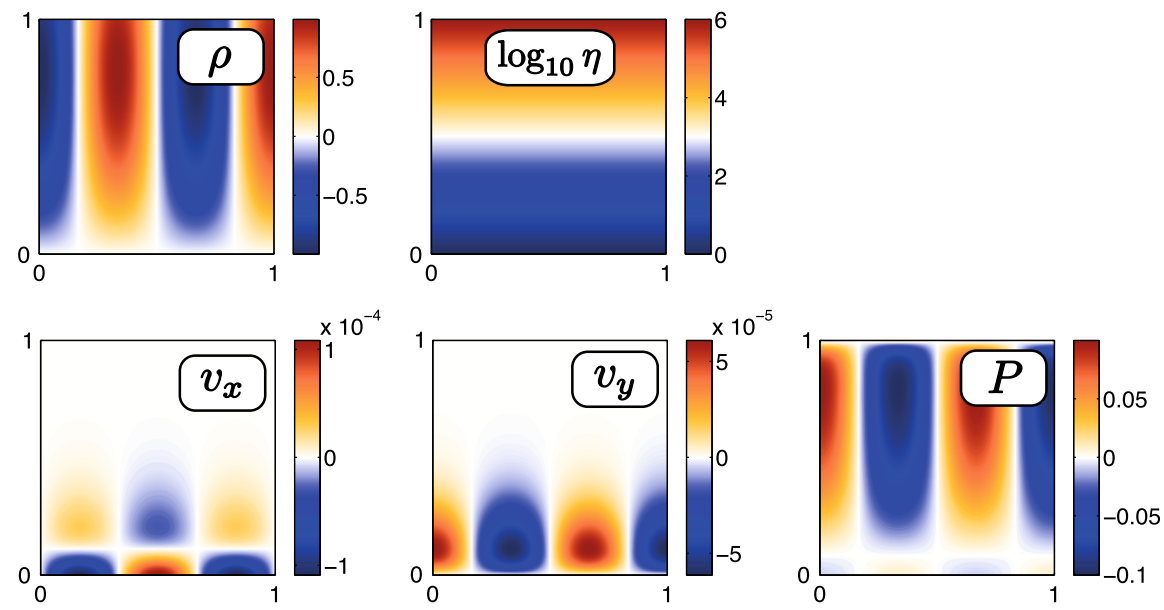

Figure 2

Density $(\rho)$ and viscosity $(\eta)$ of the SolKz benchmark as well as the analytically computed velocity $(u, v)$ and pressure $(p)$ fields
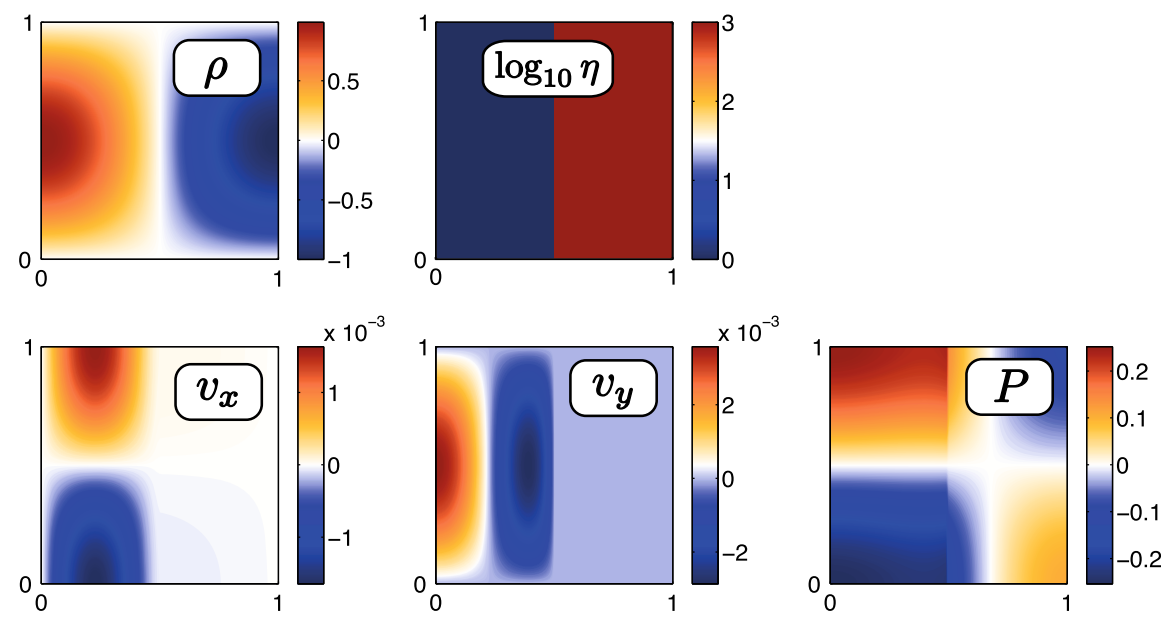

Figure 3

Velocity $(u, v)$ and pressure $(p)$ fields for the $\operatorname{SolCx}$ benchmark with chosen parameters $(\rho, \eta)$ as described in the text

\subsection{SolVI: Viscosity Jump in Two Dimensions}

In the third numerical experiment, flow is not driven by buoyancy differences, but by kinematic boundary conditions, which results in pure shear deformation. The model consists of a weak matrix with a strong circular inclusion (see Fig. 4). The analytic solution for this setup was derived in (SCHMID 2003). In this study, the model domain is defined by $[-1,1] \times[-1,1]$. The viscosity of the weak matrix and the strong inclusion is given by

$$
\eta(x, y)=\left\{\begin{array}{ccc}
1, & \text { if } & x^{2}+y^{2}>0.1 \\
10^{3}, & \text { if } & x^{2}+y^{2} \leq 0.1
\end{array} .\right.
$$

Dirichlet boundary conditions are applied to both $u, v$ and are prescribed along the entire domain boundary (their values being 1 and -1 , respectively). Dirichlet boundary velocities were computed using the analytical solution and chosen such that the system is under compression. Matlab scripts to plot the analytical solution are available from http://e-collection. library.ethz.ch/view/eth:25700.

\subsection{Error Measures}

In this study we measure the order of accuracy of the primitive variables $\mathbf{v}$ and $p$. We report errors in 

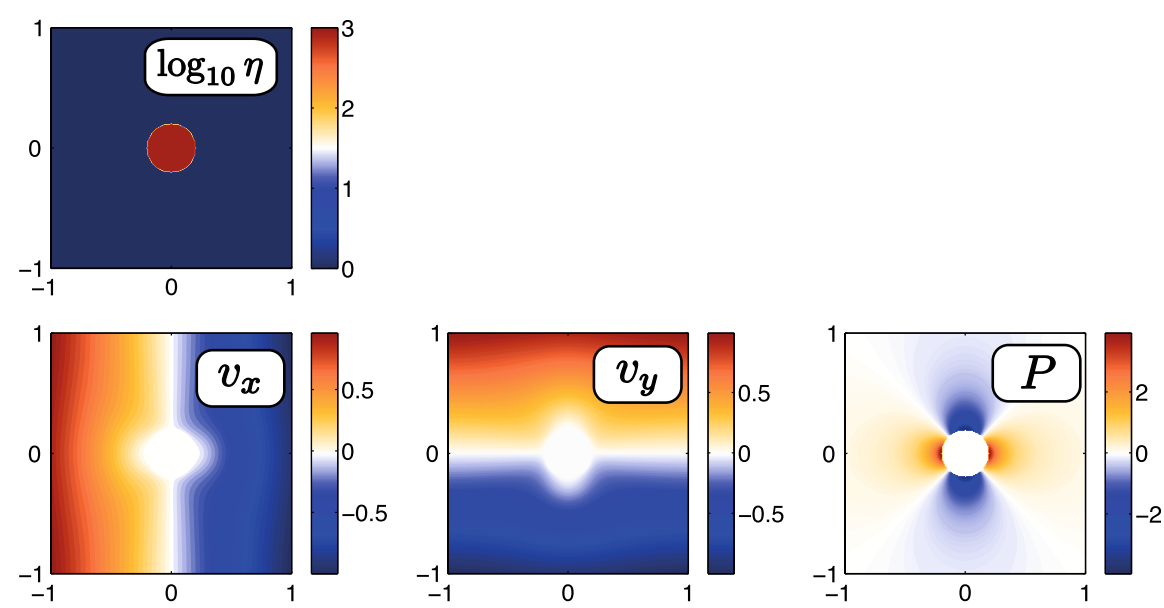

Figure 4

Velocity $(u, v)$ and pressure $(p)$ fields for the SolVI benchmark with chosen parameters $(\rho, \eta)$ as described in the text

both the $L_{1}$ and the $L_{2}$ norm. For a scalar quantity $\Psi$, the $L_{1}$ norm is computed as

$$
\|\Psi\|_{1}=\int_{V}|\Psi| d V
$$

and the $L_{2}$ norm can be computed as:

$$
\|\Psi\|_{2}=\sqrt{\int_{V} \Psi^{2} d V}
$$

For a vector quantity $\mathbf{k}=\left(\mathbf{k}_{\mathbf{1}}, \mathbf{k}_{\mathbf{2}}\right)$, the $L_{1}$ norm reads as:

$$
\|\mathbf{k}\|_{1}=\int_{V}\left(\left|k_{1}\right|+\left|k_{2}\right|\right) d V
$$

whereas the $L_{2}$ norm is defined as:

$$
\|\mathbf{k}\|_{2}=\sqrt{\int_{V}\left(k_{1}^{2}+k_{2}^{2}\right) d V} .
$$

To compute the respective norms in the case of our numerical experiments, the integrals in the above norms can be approximated by splitting them into their element-wise contributions. The element volume integral can then be easily computed by numerical integration using Gauss-Legendre quadrature. The respective $L_{1}$ and $L_{2}$ norms for pressure can be evaluated via

$$
\begin{array}{r}
\left\|e_{p}\right\|_{1}=\sum_{i}^{n_{e}} \sum_{q}^{n_{q}}\left|e_{p}\left(\mathbf{x}_{\mathbf{q}}\right)\right| \mathbf{w}_{\mathbf{q}} \operatorname{det} \mathbf{J}_{\mathbf{q}} \\
\left\|e_{p}\right\|_{2}=\sqrt{\sum_{i}^{n_{e}} \sum_{q}^{n_{q}}\left|e_{p}\left(\mathbf{x}_{\mathbf{q}}\right)\right|^{2} \mathbf{w}_{\mathbf{q}} \operatorname{det} \mathbf{J}_{\mathbf{q}}}
\end{array}
$$

where $e_{p}\left(\mathbf{x}_{\mathbf{q}}\right)=\mathbf{p}\left(\mathbf{x}_{\mathbf{q}}\right)-\mathbf{p}_{\text {analytic }}\left(\mathbf{x}_{\mathbf{q}}\right)$ is the pressure error evaluated at the $q$ th quadrature associated with the $i$ th element. $n_{e}, n_{q}$ refer to the number of elements and the number of quadrature points per element. $w_{q}, \mathbf{J}_{\mathbf{q}}$ are the quadrature weight of the Jacobian associated with point $q$. The velocity error $e_{v}$ is evaluated using the following two norms

$$
\|e v\|_{1}=\sum_{i}^{n_{e}} \sum_{q}^{n_{q}}\left[\left|e_{u}\left(\mathbf{x}_{\mathbf{q}}\right)\right|+\left|\mathbf{e}_{\mathbf{v}}\left(\mathbf{x}_{\mathbf{q}}\right)\right|\right] \mathbf{w}_{\mathbf{q}} \operatorname{det} \mathbf{J}_{\mathbf{q}},
$$

$$
\|e v\|_{2}=\sqrt{\sum_{i}^{n_{e}} \sum_{q}^{n_{q}}\left[\left|e_{u}\left(\mathbf{x}_{\mathbf{q}}\right)\right|^{2}+\left|\mathbf{e}_{\mathbf{v}}\left(\mathbf{x}_{\mathbf{q}}\right)\right|^{2}\right] \mathbf{w}_{\mathbf{q}} \operatorname{det} \mathbf{J}_{\mathbf{q}}}
$$

Analogous to the pressure error, the velocity errors are computed as $e_{u}\left(\mathbf{x}_{\mathbf{q}}\right)=\mathbf{u}\left(\mathbf{x}_{\mathbf{q}}\right)-\mathbf{u}_{\text {analytic }}\left(\mathbf{x}_{\mathbf{q}}\right)$ and $e_{v}\left(\mathbf{x}_{\mathbf{q}}\right)=\mathbf{v}\left(\mathbf{x}_{\mathbf{q}}\right)-\mathbf{v}_{\text {analytic }}\left(\mathbf{x}_{\mathbf{q}}\right)$.

We compute the different error norms for $e_{p}$ and $e v$ for a set of numerical experiments with varying resolution $h$. We expect the error norms to follow the following relationships: 


$$
\begin{gathered}
\|e v\|_{1}=C h^{r_{\mathrm{v}}} \quad\|e v\|_{2}=C h^{r_{\mathrm{v}}^{\prime}}, \\
\left\|e_{p}\right\|_{1}=C h^{r_{p}} \quad\left\|e_{p}\right\|_{2}=C h^{r_{p}^{\prime}}
\end{gathered}
$$

where $C$ is a resolution-independent constant and $r_{p}, r_{p}^{\prime}$ and $r v, r^{\prime} v$ are the convergence rates for pressure and velocity and, respectively. Using linear regression on the logarithm of the respective error norm and the resolution, we compute the convergence rates of the numerical solutions.

For $Q_{1} P_{0}$ and $Q_{2} P_{-1}$ elements, the theoretical lower bound for $r^{\prime} v$ is 2 and 3, respectively, and for $r_{p}^{\prime}$ is 1 and 2 (Elman et al. 2005). We note that when using discontinuous pressure space (e.g., $P_{0}, P_{-1}$ ), these bounds remain valid even when the viscosity is discontinuous provided that the element boundaries conform to the discontinuity.

\section{Convergence of Numerical Solutions}

For each of the tests mentioned above, we ran a set of numerical experiments using MILAMIN_VEP. We numerically measure the convergence rate of the FE-PIC solution for both $Q_{1} P_{0}$ and $Q_{2} P_{-1}$ elements using a sequence of different mesh resolutions and particle resolutions. Four different techniques to prescribe quadrature point properties were compared: direct computation of material properties to the quadrature points based on their global coordinates, arithmetic, harmonic and linear least squares interpolation. In the case of harmonic interpolation, only viscosity was interpolated using harmonic averaging, whereas density was interpolated using the arithmetic mean.

As the accuracy of finite element solutions strongly depends on if element boundaries are aligned with jumps in material properties (e.g., Deubelbeiss and Kaus 2008; Kronbichler et al. 2012; Moresi et al. 1996), we also investigate the convergence behaviour of two different sets of meshes: meshes with an even number of elements (subsequently called even meshes) and meshes with an odd number of elements (subsequently called odd meshes). In our setups, even meshes will align with viscosity jumps (in the case of the SolCx benchmark). In SolKz, this alignment is not as important, as properties are smoothly varying. In SolVI viscosity jumps occur regardless of the mesh type. However, we also report the results from those experiments for completeness. A sequence of meshes with an equal number of elements $\left(n_{\mathrm{e}}\right)$ in $x$ and $y$ was used. For $Q_{1} P_{0}$ (even) we used $n_{\mathrm{e}}=$ $M \times M$, where $M=32 \cdot 2^{d}$ with $d=[0,5]$ and for $Q_{2} P_{-1}$ (even), we used $M=16 \cdot 2^{d}$ with $d=[0,5]$. Odd meshes were defined by $M \propto 2^{d}+1$. The number of particles per element was chosen to be either $4 \times 4$ or $16 \times 16$. Particles were distributed uniformly within the element interior.

\subsection{SolKz}

The SolKz benchmark represents an idealized setup with a large, but smoothly varying viscosity contrast. In geodynamics, such a case may be found in convection studies with a stagnant lid, where no sharp changes in viscosity occur. The spatial distribution of absolute errors (computed at the quadrature points) for $\mathbf{v}$ and $p$ is shown in Fig. 5 for arithmetic and linear least squares interpolation. In both cases, the error is negligible compared to the absolute value of the variable (see Fig. 2). However, in the case of linear least squares interpolation, the error is approximately ten times smaller. Note that the error is not plotted as an element average, but at each quadrature point. For this reason, we observe error fluctuations inside each element resulting from the interpolation method. The observed error oscillations are, therefore, not numerical artefacts (we will shortly discuss this issue later).

In Fig. 6 we show $\left\|e_{\mathbf{v}}\right\|_{2}$ and $\left\|e_{p}\right\|_{2}$ for a number of grid resolutions, averaging methods and particle resolutions. In the case of $Q_{2} P_{-1}$ the expected upper bound on the order of accuracy is expected to be $\mathcal{O}\left(h^{3}\right)$ for $\|e v\|_{2}$ and $\mathcal{O}\left(h^{2}\right)$ for $\|e p\|_{2}$. This order of accuracy is only reached when directly prescribing the material properties on the quadrature points ("direct"). When using the linear least squares interpolation, we obtain the same order of accuracy for $\left\|e_{p}\right\|_{2}$, however, the order of accuracy of $\|e v\|_{2}$ decreases to values that are slightly higher than 2 . All other interpolation methods yield values of 2 and 1 for $\|e v\|_{2}$ and $\left\|e_{p}\right\|_{2}$. For $Q_{1} P_{0}$, we obtain the optimal order of accuracy independent of the interpolation 

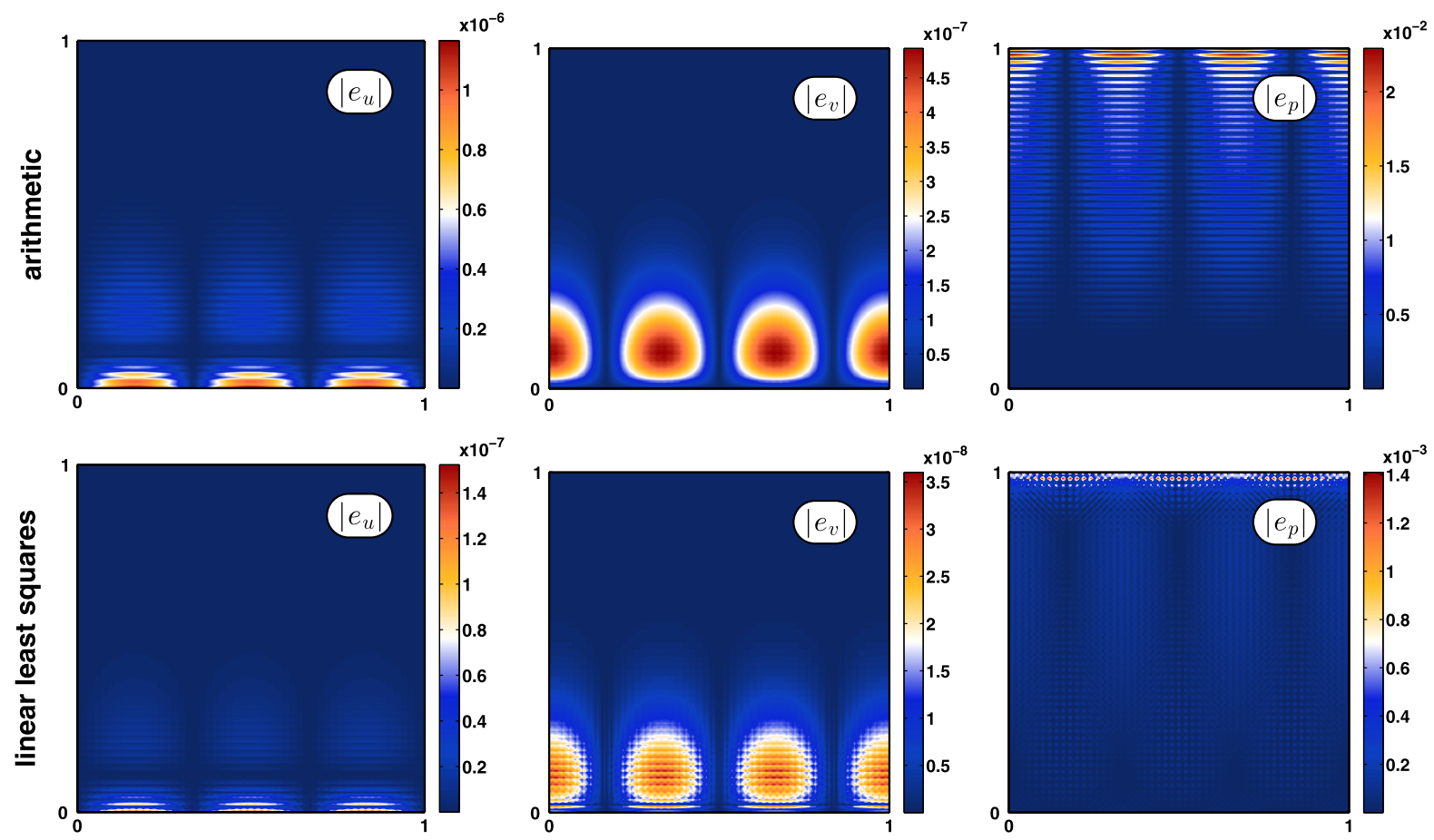

Figure 5

Spatial distribution of absolute errors for $u, v$ and $p$ for SolKz. $Q_{2} P_{-1}$ elements are used with a nodal resolution of $101 \times 101$ nodes. Material properties at the quadrature points were prescribed using a linear least squares interpolation of $16 \times 16$ particles. The top row shows the results for arithmetic interpolation, the bottom row shows the results for linear least squares interpolation. Note that the absolute errors are an order of magnitude smaller in the case of linear least squares interpolation

method used. As both density and viscosity fields are smoothly varying, the results don't differ for even or odd meshes (Table 1).

Similar to the order of accuracy, the accuracy of the numerical solution in terms of $\|e v\|_{2}$ is nearly unaffected by the used interpolation method (see Fig. 6). Arithmetic interpolation performs worst, followed by harmonic and linear least squares interpolation. Direct prescription yields the most accurate results. The number of particles per cell only slightly (if at all) influences the accuracy, its effect being the largest in the case of linear least squares interpolation, where accuracy is close to the direct method. The accuracy of $\left\|e_{p}\right\|_{2}$ is the same for all methods. Note that the pressure error is several orders of magnitude larger than the velocity error. For $Q_{2} P_{-1}$ elements, the effect of different interpolation methods on the accuracy of $\|e v\|_{2}$ is obvious (see Fig. 6). Arithmetic interpolation clearly performs worst, with harmonic interpolation producing the second worst results. Both interpolation methods are largely unaffected by the number of particles per element. Least squares interpolation performs much better in the case of a large number of particles per element. If the number of particles per element is small, the accuracy of linear least squares interpolation is comparable to harmonic interpolation. Its advantage can be clearly seen in $\left\|e_{p}\right\|_{2}$, where its accuracy is equal to the direct method (independent of the number of particles per cell). The accuracy of arithmetic and harmonic interpolation is the same in this case and errors are at least an order of magnitude higher than for the other two interpolation methods.

\subsection{SolCx with $\Delta \eta=10^{3}$}

We use SolCx to study the effect of different interpolation methods on the accuracy and convergence rate for the case of a one-dimensional viscosity jump. In Fig. 7, we plot the absolute errors of $\mathbf{v}$ and $p$. 

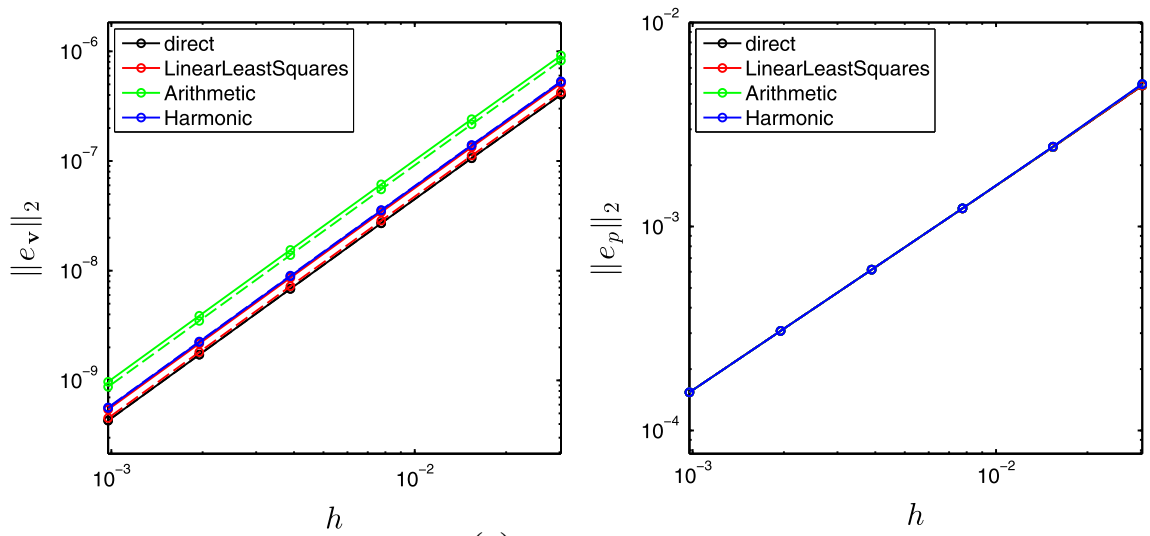

(a) $Q_{1} P_{0}$ element
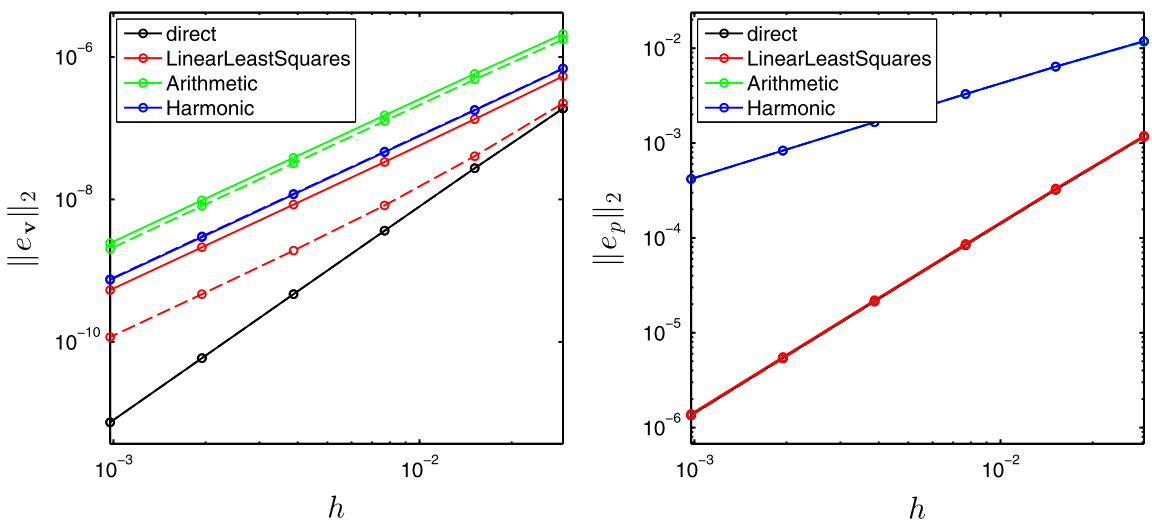

(b) $Q_{2} P_{-1}$ element

Figure 6

Velocity and pressure $L_{2}$ error norms vs. increasing resolution for the SolKz benchmark. Line colors refer to the corresponding interpolation method, with solid lines indicating the results for a small number of particles per cell $(4 \times 4)$ and dashed lines indicating the results for a large number of particles per cell $(16 \times 16)$. The slopes of the fitted lines can be found in Table 1

The upper row shows the absolute errors for an even mesh (element boundaries are aligned with the viscosity jump), whereas the lower row show the errors for an odd mesh (the viscosity jump occurs inside an element). Resolution is $101 \times 101$ nodes for the case of the even mesh and $103 \times 103$ nodes for the case of the odd mesh (As the $Q_{2} P_{-1}$ element consists of three nodes in each direction, the number of nodes is always odd. The number of nodes used in this example corresponds to $50 \times 50$ or $51 \times 51$ elements, respectively). In this example, $Q_{2} P_{1}$ elements were employed together with linear least squares interpolation using $16 \times 16$ regularly distributed particles. When element boundaries are aligned with the viscosity jump, errors are very low and negligible compared to the value of $\mathbf{v}$ and $p$ (see Fig. 3).
However, when the mesh is not aligned with the viscosity jump, errors increase significantly (by a factor of 100-1000). This has also been found in numerous other studies (KRONBICHLER et al. 2012; MoRESI et al. 1996).

In Figs. 5 and 7, we plotted the spatially varying errors for the SolKz and SolCx benchmarks. In almost all error plots, we could observe a somewhat patchy spatial distribution of the errors, in particular when the overall error was small. As previously stated, the error oscillations stem from the fact that the error is varying inside the element. $\rho$ and $\eta$ are (at best) approximated with a linear function; therefore, the interpolation error in both values varies throughout the element. The error in $u, v$ and $p$ is a result of this interpolation error. In Fig. 8, we show a zoomed 
Table 1

Orders of accuracy of the $L_{1}$ and $L_{2}$ error norms between analytical and numerical solution of the SolKz benchmark for different kinds of resolutions, elements and interpolation methods

\begin{tabular}{|c|c|c|c|c|c|c|c|c|}
\hline & \multicolumn{4}{|c|}{$Q_{1} P_{0}$, even mesh } & \multicolumn{4}{|c|}{$Q_{1} P_{0}$, odd mesh } \\
\hline & $r v$ & $r_{p}$ & $r^{\prime} v$ & $r_{p}^{\prime}$ & $r v$ & $r_{p}$ & $r^{\prime} v$ & $r_{p}^{\prime}$ \\
\hline Direct & 2.00 & 1.00 & 1.99 & 1.01 & 2.00 & 1.00 & 1.99 & 1.01 \\
\hline Arithmetic $(4 \times 4)$ & 2.00 & 1.01 & 1.99 & 1.01 & 2.00 & 1.01 & 1.99 & 1.01 \\
\hline Arithmetic $(16 \times 16)$ & 2.00 & 1.01 & 1.99 & 1.01 & 2.00 & 1.01 & 1.99 & 1.01 \\
\hline Harmonic $(4 \times 4)$ & 2.00 & 1.01 & 1.99 & 1.01 & 2.00 & 1.01 & 1.99 & 1.01 \\
\hline Harmonic $(16$ × 16) & 2.00 & 1.01 & 1.99 & 1.01 & 2.00 & 1.01 & 1.99 & 1.01 \\
\hline Linear least Squares $(4 \times 4)$ & 2.00 & 1.01 & 1.99 & 1.01 & 2.00 & 1.00 & 1.99 & 1.01 \\
\hline \multirow[t]{3}{*}{ Linear least Squares $(16 \times 16)$} & 2.00 & 1.00 & 1.99 & 1.01 & 2.00 & 1.00 & 1.99 & 1.01 \\
\hline & \multicolumn{4}{|c|}{$Q_{2} P_{-1}$, even mesh } & \multicolumn{4}{|c|}{$Q_{2} P_{-1}$, odd mesh } \\
\hline & $r v$ & $r_{p}$ & $r^{\prime} v$ & $r_{p}^{\prime}$ & $r v$ & $r_{p}$ & $r^{\prime} v$ & $r_{p}^{\prime}$ \\
\hline Direct & 3.05 & 2.00 & 2.98 & 1.99 & 3.05 & 2.00 & 2.98 & 1.99 \\
\hline Arithmetic $(4 \times 4)$ & 1.98 & 1.00 & 1.98 & 0.98 & 1.98 & 1.00 & 1.99 & 0.98 \\
\hline Arithmetic $(16 \times 16)$ & 1.99 & 1.00 & 1.99 & 0.98 & 1.99 & 1.00 & 1.99 & 0.98 \\
\hline Harmonic $(4 \times 4)$ & 1.99 & 1.02 & 2.00 & 0.98 & 1.99 & 1.02 & 2.00 & 0.99 \\
\hline Harmonic $(16 \times 16)$ & 1.99 & 1.02 & 2.00 & 0.98 & 1.99 & 1.02 & 2.00 & 0.99 \\
\hline Linear least Squares $(4 \times 4)$ & 2.00 & 2.00 & 2.03 & 1.98 & 2.00 & 2.00 & 2.02 & 1.99 \\
\hline Linear least Squares $(16 \times 16)$ & 2.09 & 2.00 & 2.21 & 1.98 & 2.09 & 2.00 & 2.20 & 1.99 \\
\hline
\end{tabular}

in part (at the viscosity jump) of the different error fields of the SolCx benchmark, where we also indicate element nodes, quadrature points and boundaries. The element-wise variability of the error fields can be clearly seen. Errors are generally smallest at the center node and increase towards the element boundaries. Interestingly, the error fields of both $\left|e_{u}\right|$ and $\left|e_{v}\right|$ have their maxima inside the element and not at the element boundaries.

In Fig. 9, we show $\|e v\|_{2}$ and $\left\|e_{p}\right\|_{2}$ for the SolCx becnhmark. Colors indicate the interpolation type, solid lines denote averaging methods using $4 \times 4$ particles per element, dashed lines denote $16 \times 16$ particles per element. Results are shown for an odd mesh. When $Q_{1} P_{0}$ elements are used, the order of accuracy is the same for all interpolation methods (see Table 2). Direct, arithmetic and linear least squares interpolation all yield the same accuracy and convergence rate. Harmonic interpolation results in smaller values of $\|e v\|_{2}$ and larger values of $\left\|e_{p}\right\|_{2}$, however, accuracies only vary marginally. In the case of $Q_{2} P_{-1}$ elements, the spread in accuracies is slightly larger. Arithmetic and linear least squares interpolation yield the same accuracy and convergence rate in $\|e v\|_{2}$. Harmonic and direct interpolation also yield the same convergence rate, with direct interpolation being most accurate. This picture is reversed for $\left\|e_{p}\right\|_{2}$, where direct interpolation yields a higher convergence rate but less accuracy. The other interpolation methods have the same order of accuracy, with linear least squares interpolation being most accurate, followed by arithmetic and harmonic interpolation.

In Table 2, we show the order of accuracy for different meshes, elements and interpolation methods. When an even mesh with $Q_{1} P_{0}$ elements is used, $\|e v\|_{1}$ and $\left\|e_{p}\right\|_{1}$ converge with $\mathcal{O}\left(h^{2}\right)$ and $\mathcal{O}(h)$ for all interpolation methods, $\|e v\|_{2}$ and $\left\|e_{p}\right\|_{2}$ converge with $\mathcal{O}\left(h^{2}\right)$ and $\mathcal{O}\left(h^{2 / 3}\right)$. Interestingly, the order of accuracy does not significantly depend on the used interpolation method. This is different for $Q_{2} P_{-1}$ elements. For an odd mesh, $L_{1}$ norms show the same order of accuracy regardless of the used interpolation scheme. For an even mesh, however, $\|e v\|_{2}$ and $\left\|e_{p}\right\|_{2}$ converge with $\mathcal{O}\left(h^{3}\right)$ and $\mathcal{O}\left(h^{2}\right)$, respectively. While the order of accuracy is reduced to two for arithmetic and harmonic interpolation, it remains larger for linear least squares interpolation. A dense particle distribution increases the convergence rate. This behaviour was already seen in the SolKz benchmark. 

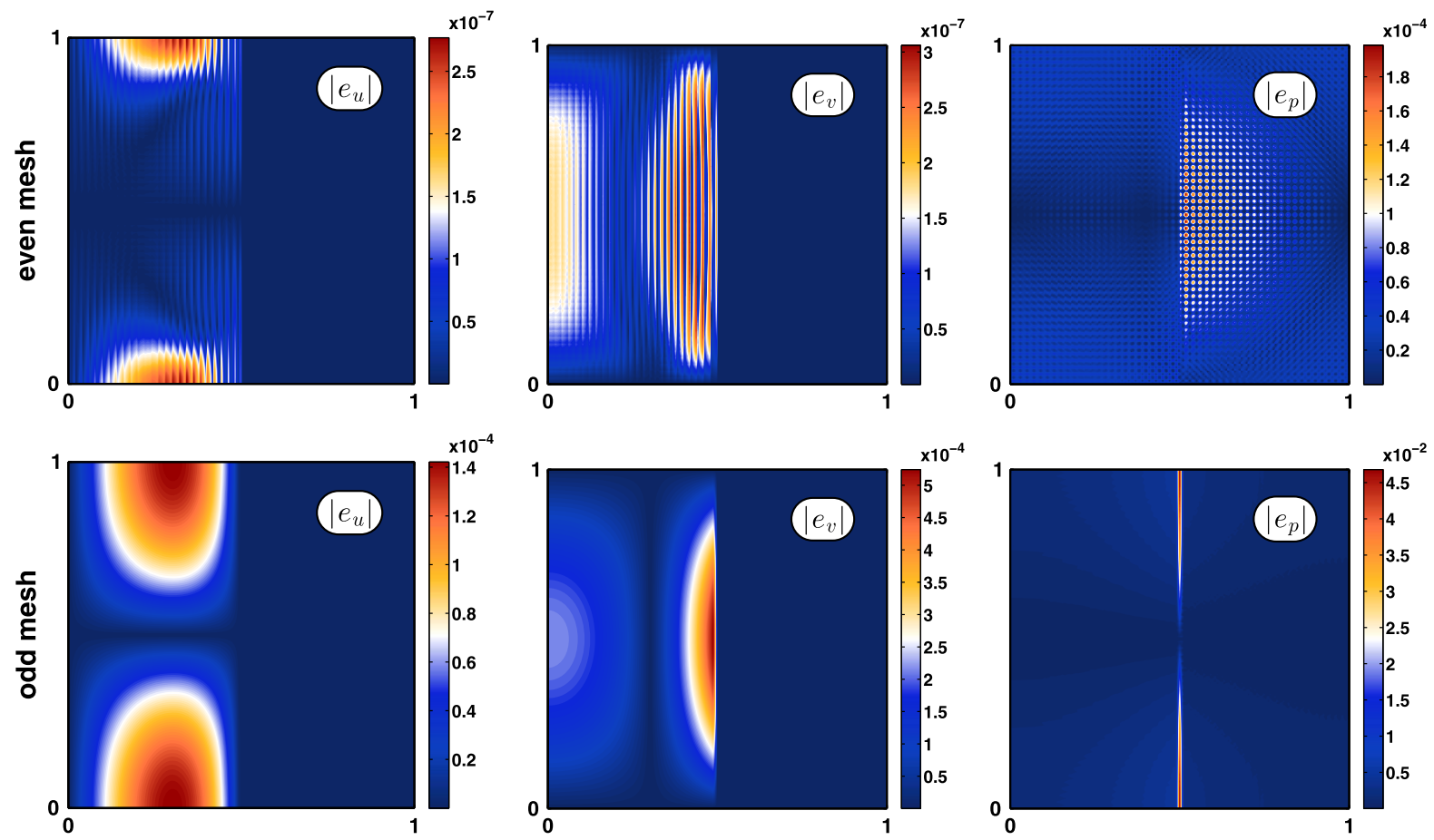

Figure 7

Spatial distribution of absolute errors for $u, v$ and $p$ for SolCx with a viscosity contrast of $\Delta \eta=10^{3}$. $Q_{2} P_{-1}$ elements are used with a nodal resolution of $101 \times 101$ nodes. Material properties at the quadrature points were prescribed using linear least squares interpolation of $16 \times 16$ particles. The top row shows the errors for an even mesh, the bottom row shows the errors for an odd mesh. Absolute errors significantly increase when an odd mesh is used with the pressure error being largest at the location of the jump

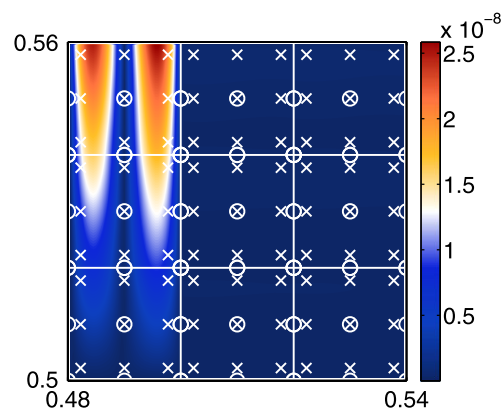

(a) $\left|e_{u}\right|$

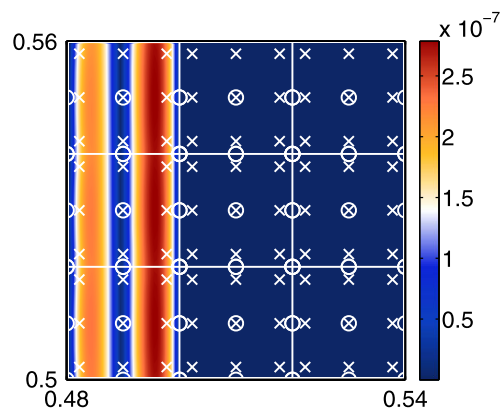

(b) $\left|e_{v}\right|$

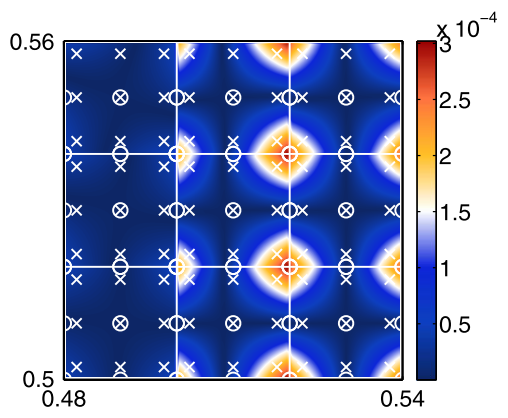

(c) $\left|e_{p}\right|$

Figure 8

Zoom on the elementwise varying error field in the SolCx benchmark for a $Q_{2} P_{-1}$ element (even mesh). White lines indicate element boundaries, white circles nodes and white crosses quadrature points. Material parameters were interpolated using linear least squares interpolation

The order of accuracy of the $L_{2}$ error norms is significantly decreased when an odd mesh is used. The order of accuracy is below $1\|e v\|_{2}$ for all interpolation methods, with arithmetic and linear least squares interpolation resulting in slightly higher convergence rates. Most important, convergence rates are on the same order as for $Q_{1} P_{0}$ elements. The same is true for the convergence rate of $\left\|e_{p}\right\|_{2}$, which is reduced to $\mathcal{O}\left(h^{1 / 2}\right)$, which is consistent with the findings of KRONBICHLER et al. (2012). 

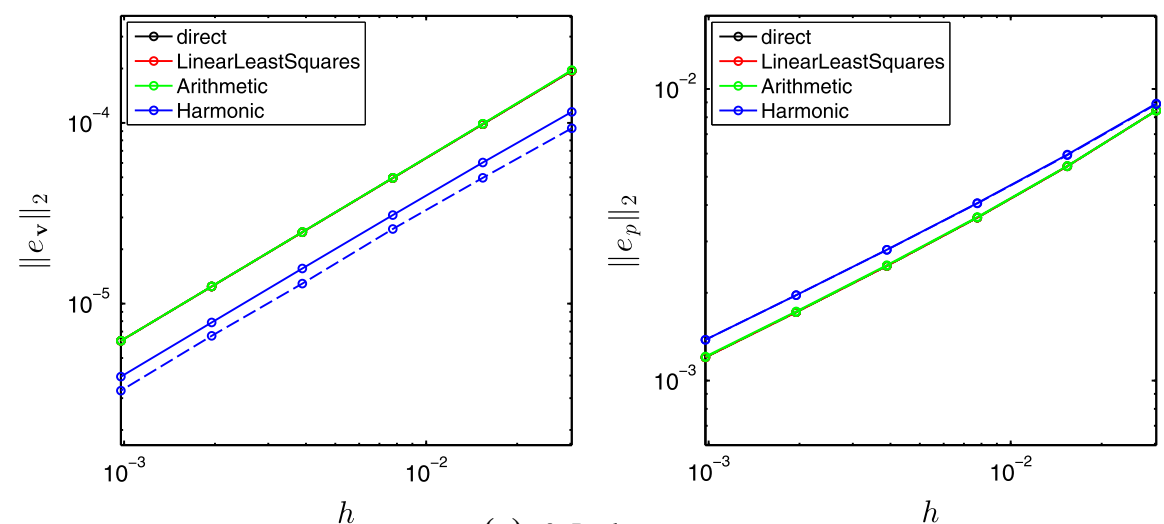

(a) $Q_{1} P_{0}$ element
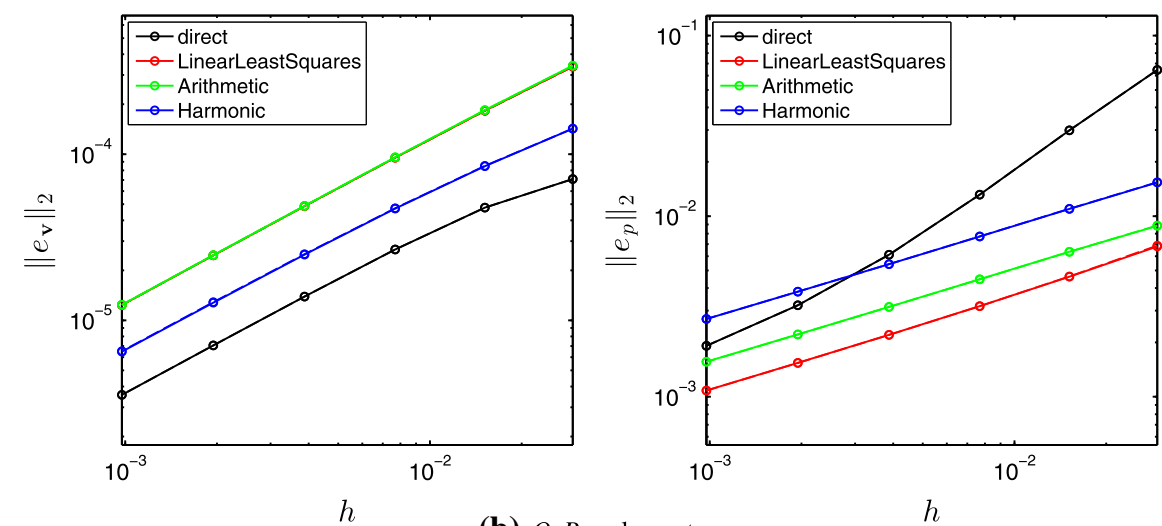

(b) $Q_{2} P_{-1}$ element

Figure 9

Velocity and pressure $L_{2}$ error norms vs. increasing resolution for the SolCx benchmark. Line colors refer to the corresponding interpolation method, with solid lines indicating the results for low number of particles per element $(4 \times 4)$ and dashed lines indicating the results for a large number of particles per element $(16 \times 16)$. The slopes of the fitted lines can be found in Table 2

\subsection{SolvI}

Of the three benchmarks used in this study, the SolVI benchmark is the most challenging one, as the viscosity jump occurs on a circular interface which cannot be captured with a structured mesh. Unlike the two other benchmarks, deformation is not driven by density differences, but by kinematic boundary conditions, the accuracy of the solution is, therefore, only dependent on the way in which we interpolate viscosity. In Fig. 10, we show the distribution of the absolute error in $\mathbf{v}$ and $p$. In this example, $Q_{2} P_{-1}$ elements are used with a nodal resolution of $201 \times 201$ nodes. Material properties are interpolated using linear least squares interpolation from $16 \times 16$ particles. As expected, errors are highest around the inclusion. The pressure oscillations at the inclusion interface and their relation to employed material parameters have been described in detail by DeuBELBEISS and KAUS (2008).

In Fig. 11, we show the $L_{2}$ norms of $e v$ and $e_{p}$ versus nodal spacing. For both element types, harmonic averaging performs best, its accuracy being largely independent of the number of particles per cell. Direct interpolation results in lower values of $\|e v\|_{2}$, but higher values of $\left\|e_{p}\right\|_{2}$. Arithmetic and linear least squares interpolation perform equally well (or less well). The accuracy of the solution is not affected by the choice of the element.

In Table 3, we report the convergence rates for different mesh types, element types and interpolation schemes. As viscosity jumps occur inside an element regardless of the mesh type used, convergence rates 
Table 2

Orders of accuracy of the $L_{1}$ and $L_{2}$ error norms between analytical and numerical solution of the SolCx with a viscosity jump of $\Delta \eta=10^{3}$ for different kinds of resolutions, elements and interpolation methods

\begin{tabular}{|c|c|c|c|c|c|c|c|c|}
\hline & \multicolumn{4}{|c|}{$Q_{1} P_{0}$, even mesh } & \multicolumn{4}{|c|}{$Q_{1} P_{0}$, odd mesh } \\
\hline & $r v$ & $r_{p}$ & $r^{\prime} v$ & $r_{p}^{\prime}$ & $r v$ & $r_{p}$ & $r^{\prime} v$ & $r_{p}^{\prime}$ \\
\hline Direct & 2.00 & 0.99 & 2.00 & 0.61 & 1.00 & 0.97 & 1.00 & 0.56 \\
\hline Arithmetic $(4 \times 4)$ & 2.00 & 0.99 & 2.00 & 0.61 & 1.01 & 0.97 & 1.00 & 0.56 \\
\hline Arithmetic $(16 \times 16)$ & 2.00 & 0.99 & 2.00 & 0.61 & 1.01 & 0.97 & 1.00 & 0.56 \\
\hline Harmonic $(4 \times 4)$ & 2.00 & 0.99 & 2.00 & 0.61 & 0.99 & 0.97 & 0.98 & 0.54 \\
\hline Harmonic $(16 \times 16)$ & 2.00 & 0.99 & 2.00 & 0.61 & 0.98 & 0.97 & 0.97 & 0.54 \\
\hline Linear least squares $(4 \times 4)$ & 2.01 & 0.99 & 2.01 & 0.61 & 1.00 & 0.97 & 1.00 & 0.56 \\
\hline \multirow[t]{3}{*}{ Linear least squares $(16 \times 16)$} & 2.01 & 0.99 & 2.01 & 0.61 & 1.00 & 0.97 & 1.00 & 0.56 \\
\hline & \multicolumn{4}{|c|}{$Q_{2} P_{-1}$, even mesh } & \multicolumn{4}{|c|}{$Q_{2} P_{-1}$, odd mesh } \\
\hline & $r v$ & $r_{p}$ & $r^{\prime} v$ & $r_{p}^{\prime}$ & $r v$ & $r_{p}$ & $r^{\prime} v$ & $r_{p}^{\prime}$ \\
\hline direct & 2.34 & 1.88 & 2.47 & 1.88 & 0.90 & 1.51 & 0.89 & 1.05 \\
\hline Arithmetic $(4 \times 4)$ & 2.01 & 1.93 & 2.00 & 1.95 & 0.98 & 0.94 & 0.98 & 0.51 \\
\hline Arithmetic $(16 \times 16)$ & 2.01 & 1.93 & 2.01 & 1.94 & 0.97 & 0.94 & 0.97 & 0.51 \\
\hline Harmonic $(4 \times 4)$ & 2.00 & 1.93 & 2.00 & 1.93 & 0.91 & 0.94 & 0.91 & 0.51 \\
\hline Harmonic $(16 \times 16)$ & 2.01 & 1.92 & 2.00 & 1.92 & 0.90 & 0.95 & 0.91 & 0.51 \\
\hline Linear least Squares $(4 \times 4)$ & 2.19 & 1.86 & 2.21 & 1.87 & 0.97 & 0.96 & 0.97 & 0.54 \\
\hline Linear least squares $(16 \times 16)$ & 2.51 & 1.88 & 2.52 & 1.88 & 0.97 & 0.96 & 0.97 & 0.54 \\
\hline
\end{tabular}
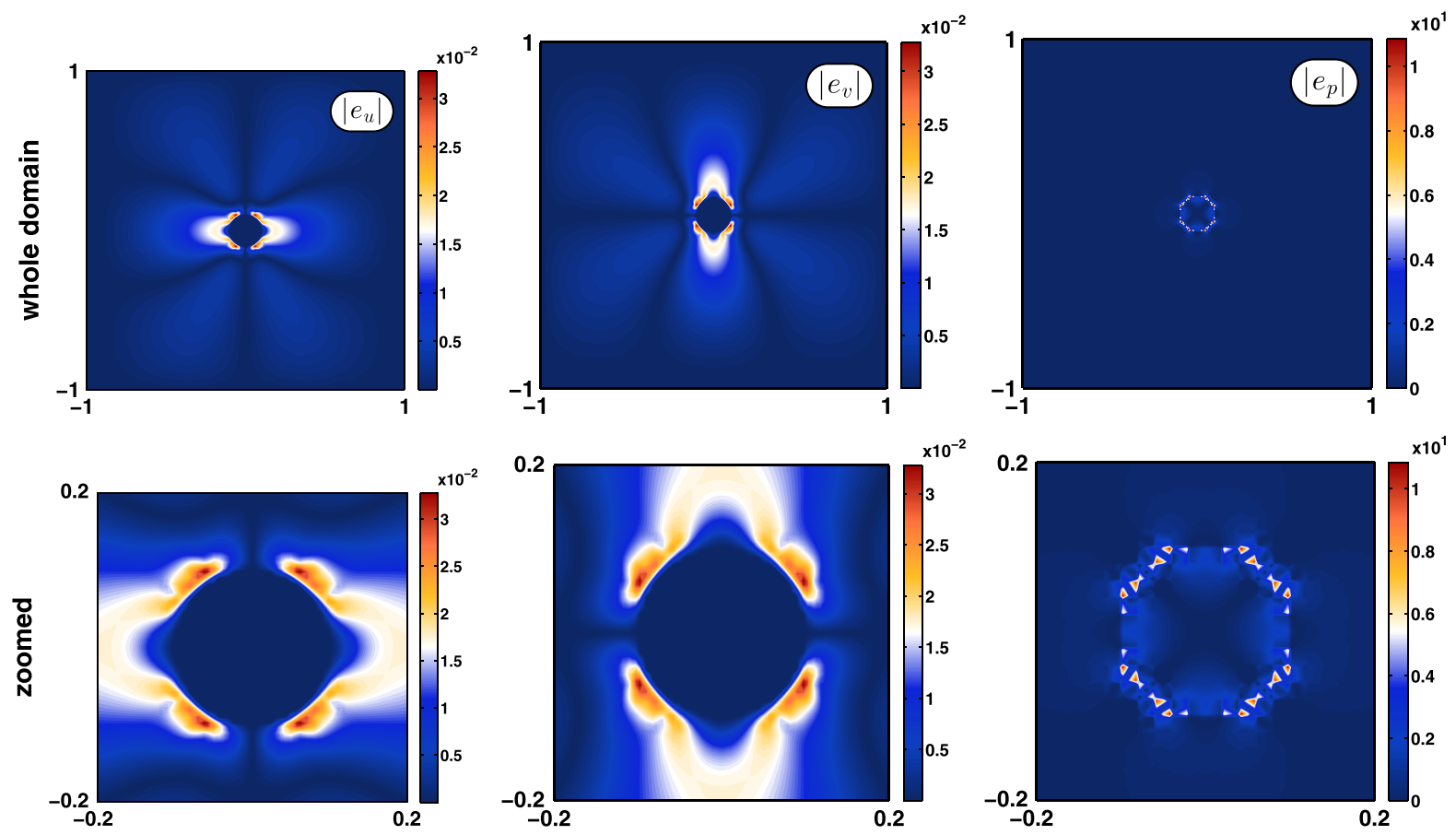

Figure 10

Spatial distribution of absolute errors for $u, v$ and $p$ for SolVI with a viscosity contrast of $\Delta \eta=10^{3}$. $Q_{2} P_{-1}$ elements are used with a nodal resolution of $201 \times 201$ nodes. Material properties at the quadrature points were prescribed using linear least squares interpolation of $16 \times 16$ particles. The top row shows the errors in the whole domain, the bottom row shows the errors in a zoomed in region. The pressure oscillations 

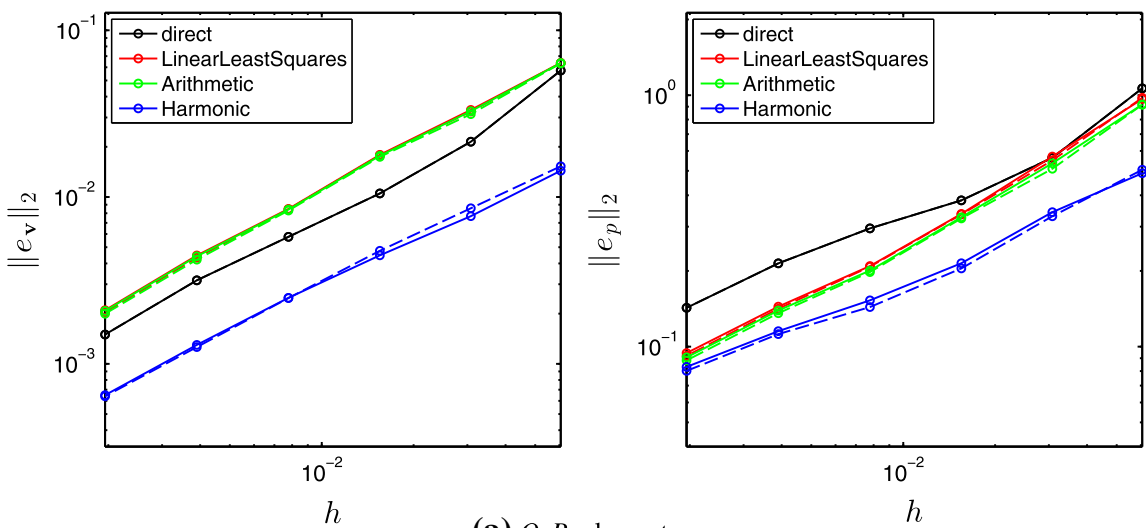

(a) $Q_{1} P_{0}$ element

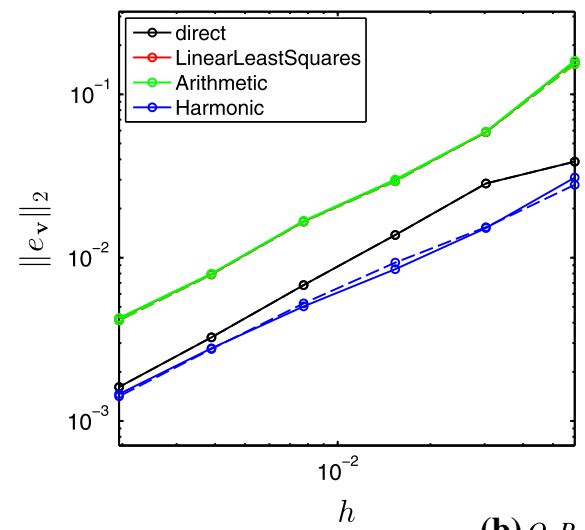

(b) $Q_{2} P_{-1}$ element

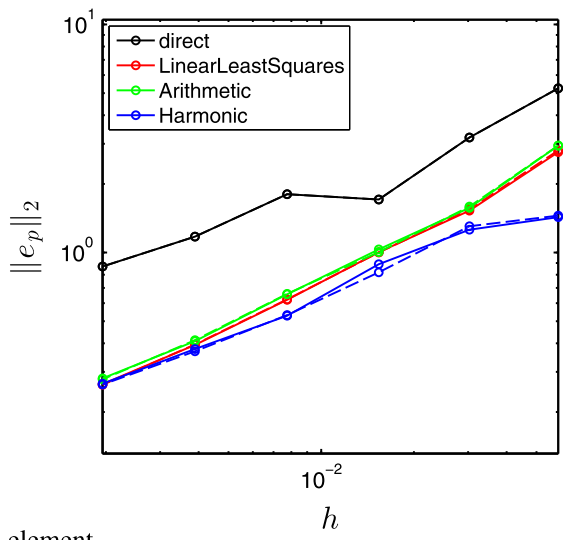

Figure 11

Velocity and pressure $L_{2}$ error norms vs. increasing resolution for the SolVI benchmark. Line colors refer to the corresponding interpolation method, with solid lines indicating the results for a small number of particles per cell $(4 \times 4)$ and dashed lines indicating the results for a large number of particles per cell $(16 \times 16)$. The slopes of the fitted lines can be found in Table 3

do not differ significantly for even or odd meshes. Convergence rates of $\|e v\|_{1}$ and $\left\|e_{p}\right\|_{1}$ are not significantly affected by the used element type and are both of $\mathcal{O}(h)$. The convergence rate of $\|e v\|_{2}$ is also of $\mathcal{O}(h)$, with arithmetic and linear least squares interpolation having the largest convergence rates. The convergence rates for $\left\|e_{p}\right\|_{2}$ range between $\frac{1}{2}$ and $\frac{3}{4}$. Again, the largest convergence rates are obtained when using arithmetic or linear least squares interpolation.

As described in (MACKINNON and CAREY 1987), the cause for the loss of accuracy when inter-element jumps are introduced is simply because the interpolants used for velocity and pressure do not permit inter-element jumps to be represented. Thus, whilst PIC type methods permit large deformation processes to be modeled, using non-body fitted finite elements will naturally result in low order accuracy methods. In order to increase the order of accuracy, the interpolants within the those element containing jumps in viscosity must be modified such that jumps in strain-rate and pressure can be described. To maintain the benefits, we sought by using the FE-PIC approach, one could consider employ methods which only locally modify the basis functions with supports, which intersect the viscosity jumps such as; XFEM (BelytschKo et al. 2001; Fries 2009; Fries and BelytschKo 2010; Legrain et al. 2008; Sussman and FAtemi 1999; ZlotNiK and Dìez 2009) immersed interface method (Li and Iто 2006), partition of unity and multi-scale enrichment methods (Albers 2012; Chessa et al. 2003; Dolbow and Devan 2004; 
Table 3

Orders of accuracy of the $L_{1}$ and $L_{2}$ error norms between analytical and numerical solution of the SolVI with a viscosity jump of $\Delta y=10^{3}$ for different kinds of resolutions, elements and interpolation methods

\begin{tabular}{|c|c|c|c|c|c|c|c|c|}
\hline & \multicolumn{4}{|c|}{$Q_{1} P_{0}$, even mesh } & \multicolumn{4}{|c|}{$Q_{1} P_{0}$, odd mesh } \\
\hline & $r v$ & $r_{p}$ & $r^{\prime} v$ & $r_{p}^{\prime}$ & $r v$ & $r_{p}$ & $r^{\prime} v$ & $r_{p}^{\prime}$ \\
\hline Direct & 1.02 & 0.97 & 1.01 & 0.60 & 1.03 & 0.93 & 1.02 & 0.55 \\
\hline Arithmetic $(4 \times 4)$ & 1.04 & 0.96 & 1.02 & 0.65 & 1.00 & 0.96 & 0.99 & 0.67 \\
\hline Arithmetic $(16 \times 16)$ & 1.02 & 0.95 & 1.01 & 0.64 & 1.01 & 0.97 & 1.00 & 0.67 \\
\hline Harmonic $(4 \times 4)$ & 0.94 & 0.83 & 0.93 & 0.47 & 0.90 & 0.84 & 0.89 & 0.52 \\
\hline Harmonic $(16 \times 16)$ & 0.95 & 0.85 & 0.94 & 0.47 & 0.94 & 0.87 & 0.93 & 0.53 \\
\hline Linear least squares $(4 \times 4)$ & 1.04 & 0.95 & 1.02 & 0.66 & 1.00 & 0.96 & 0.99 & 0.67 \\
\hline \multirow[t]{3}{*}{ Linear least squares $(16 \times 16)$} & 1.03 & 0.95 & 1.01 & 0.64 & 1.01 & 0.97 & 1.00 & 0.68 \\
\hline & \multicolumn{4}{|c|}{$Q_{2} P_{-1}$, even mesh } & \multicolumn{4}{|c|}{$Q_{2} P_{-1}$, odd mesh } \\
\hline & $r v$ & $r_{p}$ & $r^{\prime} v$ & $r_{p}^{\prime}$ & $r v$ & $r_{p}$ & $r^{\prime} v$ & $r_{p}^{\prime}$ \\
\hline Direct & 1.02 & 0.86 & 1.01 & 0.41 & 0.98 & 0.91 & 0.97 & 0.50 \\
\hline Arithmetic $(4 \times 4)$ & 1.06 & 1.04 & 1.03 & 0.69 & 1.06 & 1.04 & 1.03 & 0.68 \\
\hline Arithmetic $(16 \times 16)$ & 1.06 & 1.04 & 1.03 & 0.69 & 1.06 & 1.04 & 1.03 & 0.68 \\
\hline Harmonic $(4 \times 4)$ & 0.89 & 0.83 & 0.87 & 0.50 & 0.89 & 0.84 & 0.87 & 0.53 \\
\hline Harmonic $(16 \times 16)$ & 0.87 & 0.83 & 0.85 & 0.50 & 0.88 & 0.85 & 0.86 & 0.53 \\
\hline Linear least squares $(4 \times 4)$ & 1.06 & 1.03 & 1.03 & 0.69 & 1.06 & 1.03 & 1.03 & 0.68 \\
\hline Linear least squares $(16 \times 16)$ & 1.06 & 1.04 & 1.03 & 0.68 & 1.06 & 1.03 & 1.03 & 0.69 \\
\hline
\end{tabular}

Dolbow et al. 2000; Fish and YuAn 2005; Mourad et al. 2007). We hope that in the near future, such methods development in the engineering community which are specifically designed to capture subelement constitutive behaviour will be examined in the context of geodynamic applications.

By noting that the loss of accuracy can be attributed to the inability to represent discontinuous pressure and strain rate fields within an element, we conclude that all hybrid FE-PIC methods will exhibit similar characteristics to the results we have presented here. Moresi et al. (2003) used the particle locations as the quadrature points and computed the respective weights using Voronoi diagrams. We remark that this approach is elegant in that it eliminates the need to construct an interpolant to project material properties onto quadrature points, nevertheless, the underlying loss of order of accuracy we document here will still be present in this type of "interpolate free" PIC method.

In contrast to the moving integration point method of MoResi et al. (2003), our approach is easily extensible to higher order elements as the task of constructing an approximate representation of the material properties is independent of the quadrature scheme used to evaluate the weak form. Furthermore, the least squares projection approach described here is cheaper to construct as it avoids the need to compute an approximate Voronoi diagram.

In contrast to the discontinuous viscosity case, if the viscosity (and density) fields are smoothly varying (as in the SolKz benchmark), the convergence and accuracy are significantly improved using the new least square interpolation scheme presented here. Smoothly varying viscosity and density fields are commonly encountered in mantle convection models where viscosity and density are temperature dependent and pressure dependent. For such modeling scenarios, the presented interpolation method combined with a higher order element yields more accurate results for lower resolutions. The additional effort to perform a slightly more sophisticated interpolation from the particles to the quadrature points pays off in this case.

\section{Conclusions}

In this study, we investigated the discretization errors and the convergence properties of the hybrid 
FE-PIC (finite element particle-in-cell) method. We introduced a new interpolation scheme, which is relatively cheap and that takes into account the variation of material properties throughout the element. The analysis was carried out by comparing analytical and numerical solutions of three idealized model setups. The different model setups test the ability of an interpolation scheme to deal with either smoothly varying, one-dimensional discontinuous and twodimensional discontinuous viscosity fields. We tested three different interpolation schemes: arithmetic, harmonic and linear least squares interpolation together with two different element types: $Q_{1} P_{0}$ and $Q_{2} P_{-1}$. We find that in the case of smoothly varying viscosities, the linear least squares interpolation together with the $Q_{2} P_{-1}$ element results in optimal convergence and high accuracy. This type of numerical scheme is, therefore, optimal for mantlelithosphere simulations with continuously varying viscosity fields.

In conclusion, the additional cost of using linear least squares interpolation together with a high-order element does not pay off when the viscosity field exhibits sharp changes. Therefore, a linear element would be sufficient for those purposes. However, it is known that low-order elements can sometimes exhibit numerical artefacts in incompressible problems, where velocities are significantly underestimated [also known as 'locking', see, e.g., Popov and SовоLEV (2008)]. Furthermore, low order elements are not very accurate [see the results for $Q_{1} P_{0}$ in this study and the discussion in Kronbichler et al. (2012)] and, in particular, $Q_{1} P_{0}$-elements are known to sometimes show some kind of checkerboard pattern in the pressure field (BATHE 1982), which requires some kind of smoothing of the pressure field (e.g., Braun et al. 2008). More work is required to obtain a stable and accurate low order (linear) element for Stokes flow problems.

\section{Acknowledgments}

The comments by S. Buiter and an anonymous reviewer are greatly appreciated. The research leading to these results has received funding from Crystal2Plate, an FP7-funded Marie Curie Action under Grant agreement number PITN-GA-2008215353. M.T. has received support from SNF Grant 200021-143299 and B.K. was supported by ERC Starting Grant 258830.

\section{REFERENCES}

Albers M (2000) A Local Mesh Refinement Multigrid Method for 3-D Convection Problems with Strongly Variable Viscosity. Journal of Computational Physics 160(1):126-150.

Ausas RF, Buscaglia GC, Idelsohn SR (2012) A new enrichment space for the treatment of discontinuous pressures in multi-fluid flows. International Journal for Numerical Methods in Fluids 70(7):829-850.

Bathe K (1982) Finite Element Procedures in Engineering Analysis. Prentice-Hall Civil Engineering and Engineering Mechanics Series, Prentice-Hall International.

Belytschko T, Moës N, Usui S, Parimi C (2001) Arbitrary discontinuities in finite elements. International Journal for Numerical Methods in Engineering 50(4):993-1013.

Braun J, Thieulot C, Fullsack P, DeKool M (2008) Douar: A new three-dimensional creeping flow numerical model for the solution of geological problems. Physics of the Earth and Planetary Interiors.

Chessa J, Wang H, Belytschko T (2003) On the construction of blending elements for local partition of unity enriched finite elements. International Journal for Numerical Methods in Engineering 57(7):1015-1038.

Cockburn B, Shu CW (1998) The Runge-Kutta discontinuous Galerkin method for conservation laws $V$ : multidimensional systems. Journal of Computational Physics 141(2):199-224.

Cockburn B, Karniadakis G, Shu CW (2000) The development of discontinuous Galerkin methods. In: Cockburn B, Karniadakis G, Shu CW (eds) Discontinuous Galerkin Methods, Lecture Notes in Computational Science and Engineering, vol 11, Springer, Berlin Heidelberg, pp 3-50.

Dabrowski M, Krotkiewski M, Schmid D (2008) MILAMIN: MATLAB-based finite element method solver for large problems. Geochemistry Geophysics Geosystems 9:Q04,030.

Deubelbeiss Y, Kaus BJP (2008) Comparison of Eulerian and Lagrangian numerical techniques for the Stokes equations in the presence of strongly varying viscosity. Physics of the Earth and Planetary Interiors 171:92-111.

Dolbow J, Devan A (2004) Enrichment of enhanced assumed strain approximations for representing strong discontinuities: addressing volumetric incompressibility and the discontinuous patch test. International journal for numerical methods in engineering 59(1):47-67.

Dolbow J, Mö̈s N, BelytschKo T (2000) Discontinuous enrichment in finite elements with a partition of unity method. Finite elements in analysis and design 36(3):235-260.

Duretz T, May DA, Gerya TV, Tackley PJ (2011) Discretization errors and free surface stabilization in the finite difference and marker-in-cell method for applied geodynamics: A numerical study. Geochemistry Geophysiscs Geosystems 12(7).

Elman HC, Silvester DJ, Wathen AJ (2005) Finite Elements and Fast Iterative Solvers: With Applications in Incompressible Fluid Dynamics. Numerical Mathematics and Scientific Computation, OUP Oxford. 
Fish J, YUAN Z (2005) Multiscale enrichment based on partition of unity. International Journal for Numerical Methods in Engineering 62(10):1341-1359.

FRIES TP (2009) The intrinsic XFEM for two-fluid flows. International Journal for Numerical Methods in Fluids 60(4):437-471.

FRIES TP, BELYTSCHKO T (2010) The extended/generalized finite element method: an overview of the method and its applications. International Journal for Numerical Methods in Engineering 84(3):253-304.

GERYA TV (2003) Characteristics-based marker-in-cell method with conservative finite-differences schemes for modeling geological flows with strongly variable transport properties. Physics of the Earth and Planetary Interiors 140:293-318.

Kaus BJP, PodLadChIKov YY (2001) Forward and reverse modeling of the three-dimensional viscous Rayleigh-Taylor instability. Geophys Res Lett.

Kaus BJP, MüHlhaus H, May DA (2010) A stabilization algorithm for geodynamic numerical simulations with a free surface. Physics of the Earth and Planetary Interiors 181:12-20.

Kronbichler M, Heister T, Bangerth W (2012) High accuracy mantle convection simulation through modern numerical methods. Geophysical Journal International 191:12-29.

Legrain G, MoËs N, Huerta A (2008) Stability of incompressible formulations enriched with x-fem. Computer Methods in Applied Mechanics and Engineering 197(21):1835-1849.

LeVeque RJ (2002) Finite volume methods for hyperbolic problems, vol 31. Cambridge university press.

Li Z, Iто K (2006) The immersed interface method: numerical solutions of PDEs involving interfaces and irregular domains, vol 33. Siam.

Mackinnon R, CAREy G (1987) Treatment of material discontinuities in finite element computations. International journal for numerical methods in engineering 24(2):393-417.

MAy DA, Moresi L (2008) Preconditioned iterative methods for stokes flow problems arising in computational geodynamics. Physics of the Earth and Planetary Interiors 171(1):33-47.

Moresi L, Zhong S, GuRnis M (1996) The accuracy of finite element solutions of Stokes's flow with strongly varying viscosity. Physics of the Earth and Planetary Interiors 97(1-4):83-94.

Moresi L, Dufour F, Mühlhaus HB (2003) A lagrangian integration point finite element method for large deformation modeling of viscoelastic geomaterials. Journal of Computational Physics 184(2):476-497.

Moresi L, Quenette S, Lemiale V, Meriaux C (2007) Computational approaches to studying non-linear dynamics of the crust and mantle. Physics of the Earth and Planetary Interiors 163:69-82.

Mourad HM, Dolbow J, Harari I (2007) A bubble-stabilized finite element method for dirichlet constraints on embedded interfaces. International journal for numerical methods in engineering 69(4):772-793.

Osher S, FEDKIw R (2003) Level set methods and dynamic implicit surfaces, vol 153. Springer.
Poliakov A, Podladchikov YY (1992) Diapirism and topography. Geophysical Journal International 109(3):553-564.

Popov A, Sobolev S (2008) SLIM3D: A tool for three-dimensional thermomechanical modeling of lithospheric deformation with elasto-visco-plastic rheology. Physics of the Earth and Planetary Interiors.

QuinQUis MET, BuITER SJH (2013) Testing the effects of the numerical implementation of water migration on models of subduction dynamics. Solid Earth Discussions 5(2), pp. 1771-1815, doi 10.5194/sed-5-1771-2013, URL http://www. solid-earth-discuss.net/5/1771/2013/

Revenaugh J, Parsons B (1987) Dynamic topography and gravity anomalies for fluid layers whose viscosity varies exponentially with depth. Geophysical Journal International 90(2):349-368.

Schmeling H, Babeyko A, Enns A, Faccenna C, Funiciello F, Gerya TV, Golabek G, Grigull S, Kaus BJP, Morra G, SchMalholz S, van Hunen J (2008) A benchmark comparison of spontaneous subduction models-Towards a free surface. Physics of the Earth and Planetary Interiors 171:198-223.

Schmid D (2003) Analytical solutions for deformable elliptical inclusions in general shear. Geophysical Journal International 155:269-288.

SETHIAN JA (1999) Level set methods and fast marching methods: evolving interfaces in computational geometry, fluid mechanics, computer vision, and materials science, vol 3. Cambridge university press.

Sussman M, Fatemi E (1999) An efficient, interface-preserving level set redistancing algorithm and its application to interfacial incompressible fluid flow. SIAM Journal on Scientific Computing 20(4):1165-1191.

TACKLEY P (2000) Self-consistent generation of tectonic plates in time-dependent, threedimensional mantle convection simulations, part 1: Pseudoplastic yielding. Geochemistry Geophysics Geosystems 1.

TACKLEY P, King S (2003) Testing the tracer ratio method for modeling active compositional fields in mantle convection simulations. Geochemistry Geophysics Geosystems 4(4). doi:10. 1029/2001GC000214

TROMPERT RA, HANSEN U (1996) The application of a finite volume multigrid method to three-dimensional flow problems in a highly viscous fluid with a variable viscosity. Geophysical \& Astrophysical Fluid Dynamics 83(3-4):261-291.

VAn KeKen P, King S, Schmeling H (1997) A comparison of methods for the modeling of thermochemical convection. Journal of Geophysical Research 102:22477-22495.

ZHong S (1996) Analytic solutions for Stokes' flow with lateral variations in viscosity. Geophysical Journal International 124(1):18-28.

Zienkiewicz OC, TAYLOR RL, ZHU JZ (2005) The Finite Element Method: Its Basis and Fundamentals, 6th edn. Its Basis and Fundamentals, Butterworth-Heinemann, Oxford.

ZlotNik S, DíEZ P (2009) Hierarchical $x$-fem for $n$-phase flow $(n>2)$. Computer Methods in Applied Mechanics and Engineering 198(30):2329-2338. 